\title{
Consistent large-eddy simulation of a temporal mixing layer laden with evaporating drops. Part 2. A posteriori modelling
}

\author{
By ANTHONY LEBOISSETIER, NORA OKONG'O \\ AND JOSETTE BELLANं \\ Jet Propulsion Laboratory, California Institute of Technology, Pasadena, CA 91109-8099, USA
}

(Received 26 January 2004 and in revised form 24 August 2004)

Large-eddy simulation (LES) is conducted of a three-dimensional temporal mixing layer whose lower stream is initially laden with liquid drops which may evaporate during the simulation. The gas-phase equations are written in an Eulerian frame for two perfect gas species (carrier gas and vapour emanating from the drops), while the liquid-phase equations are written in a Lagrangian frame. The effect of drop evaporation on the gas phase is considered through mass, species, momentum and energy source terms. The drop evolution is modelled using physical drops, or using computational drops to represent the physical drops. Simulations are performed using various LES models previously assessed on a database obtained from direct numerical simulations (DNS). These LES models are for: (i) the subgrid-scale (SGS) fluxes and (ii) the filtered source terms (FSTs) based on computational drops. The LES, which are compared to filtered-and-coarsened (FC) DNS results at the coarser LES grid, are conducted with 64 times fewer grid points than the DNS, and up to 64 times fewer computational than physical drops. It is found that both constantcoefficient and dynamic Smagorinsky SGS-flux models, though numerically stable, are overly dissipative and damp generated small-resolved-scale (SRS) turbulent structures. Although the global growth and mixing predictions of LES using Smagorinsky models are in good agreement with the FC-DNS, the spatial distributions of the drops differ significantly. In contrast, the constant-coefficient scale-similarity model and the dynamic gradient model perform well in predicting most flow features, with the latter model having the advantage of not requiring a priori calibration of the model coefficient. The ability of the dynamic models to determine the model coefficient during LES is found to be essential since the constant-coefficient gradient model, although more accurate than the Smagorinsky model, is not consistently numerically stable despite using DNS-calibrated coefficients. With accurate SGS-flux models, namely scale-similarity and dynamic gradient, the FST model allows up to a 32-fold reduction in computational drops compared to the number of physical drops, without degradation of accuracy; a 64-fold reduction leads to a slight decrease in accuracy.

\section{Introduction}

Two-phase (TP) flows involving evaporating liquid drops being transported by a turbulent flow occur in many applications of practical interest, such as household

$\dagger$ Author to whom correspondence should be addressed: Josette.Bellan@jpl.nasa.gov 
cleaning products, pharmaceutical inhalers, office printers, gas turbine engines and spray-chemical-conversion reactors. In many of these situations, good atomization means that the size of the drops should be minimized. When the size of the drops is much smaller than the Kolmogorov scale and the volumetric loading is small $\left(\simeq 10^{-3}\right)$, Boivin, Simonin \& Squires (1998) have shown that the drops can be treated as point sources of mass, momentum and energy into the gas phase. In such situations, TP direct numerical simulation (DNS) can be conducted with the resolution adequate for single-phase (SP) flow and the meaning of DNS for such TP flows is thus identical to that of SP flows in that all turbulence scales of the gaseous flow are resolved. When the mass loading is small, the effect of the flow field on the drops can be modelled through one-way coupling, as the flow influences the drops but their presence does not affect the flow. Due to the large ratio of liquid density to carrier-gas density, the mass loading may however be significant, causing the drops to considerably influence the flow, and two-way coupling may prevail. Recent TP DNS for such flows, for isotropic turbulence, include those of Boivin et al. (1998) and Mashayek \& Jaberi (1999) for solid particles without phase change and those of Mashayek (1998a) and Réveillon \& Vervisch (2000) for evaporating drops. Other DNS with evaporating drops have been performed by Mashayek (1998b) for homogeneous shear flow and by Miller \& Bellan $(1999,2000)$ and Okong'o \& Bellan (2004) (Part 1) for temporal mixing layers. The study of the irreversible entropy production (i.e. dissipation) using the DNS database in Part 1 suggested caution in extrapolating solid particle results to the realm of evaporating drops, as most of the dissipation was of thermodynamic and evaporative, rather than dynamic, origin.

On the other hand, large-eddy simulation (LES) is less computationally demanding than DNS. A coarser grid can be used in LES, since only the large scales are resolved while the subgrid scales (SGSs) are modelled, and a smaller number of 'computational' drops may be used to represent the full number of physical drops. The LES gas-phase equations are obtained from the DNS set by spatial filtering and through this process, in a compressible multi-species formulation, SGS momentum, energy and species fluxes arise from the convective terms (the SGS fluxes appearing in the momentum equation are the SGS stresses). The SGS fluxes and the filtered source terms (FSTs) in the LES equations cannot be computed from the resolved filtered flow field, and must instead be modelled. Most LES of TP flows have neglected direct SGS effects on the particles; such an approach was justified by Wang \& Squires (1996), who found SGS effects, included by modifying the gas-phase velocity felt by the particles, to be negligible. Recent TP LES, including those by Boivin, Simonin \& Squires (2000), Deutsch \& Simonin (1991), Simonin, Deutsch \& Boivin (1995), Uijttewaal \& Oliemans (1996), Wang \& Squires (1996) and Yamamoto et al. (2001), have been for an incompressible gas phase laden with small solid particles with one-way coupling (Boivin et al. 2000 and Yamamoto et al. 2001 also considered two-way coupling). These studies used physical or computational particles whose evolution was entirely governed by the resolved flow field. Because the SGS modelling in these LES was confined to the gas phase, SGS-flux models for incompressible SP flow could be used; these LES used constant-coefficient or dynamic-coefficient Smagorinsky (see Smagorinksy 1993) SGS-flux modelling, which is based on gradient diffusion with an eddy viscosity coefficient. The Smagorinsky model was also used in the TP LES of solid particles by Yuu, Ueno \& Umekage (2001); in their incompressible flow, the particles were assumed monodisperse, and the SGS-flux of drop number density appearing in the FSTs was also modelled using the gradient-diffusion concept. 
However, for the situation of evaporating drops, where two additional equations (for energy and species mass) are needed, a compressible formulation must be employed to account for the density change in the gas phase. Although several SP studies have considered SGS models for the energy equation, the type of SGS model depends on the form of the energy equation used (see Erlebacher et al. 1992; Fureby 1996; Martin, Piomelli \& Candler 2000); typically, the same formulation is used for modelling the energy and species-mass SGS fluxes. SGS TP flow models that treated the modelling of the drop contribution were presented by Miller \& Bellan (2000) and Okong'o \& Bellan (2000), but in these studies the size of the drop ensemble was not reduced nor were the FSTs, representing the effect of the drops on the flow field, considered. Such considerations have been addressed by Sankaran \& Menon (2002) for TP LES of swirling flow with evaporating drops, wherein the SGS effects on the computational drops were modelled using the same approach as Wang \& Squires (1996), that is, by modifying the flow velocity felt by the particles. Sankaran \& Menon (2002) used the Smagorinsky eddy-viscosity approach to model the SGS fluxes in the momentum equation, as well as in the species and energy equations; however, they did not perform comparisons to experiments or DNS.

In the present paper, we perform LES for the same conditions as the temporal mixing layer TP DNS of Part 1, using that DNS database for rigorous a posteriori assessment of the SGS-flux and FST models that were analysed a priori in Part 1. The a priori study of these models led to the conclusion that models developed for SP LES were suitable for the SGS fluxes. While some of the FST models of Part 1 did incorporate direct SGS effects on the drops, the additional complexity introduced by modelling direct SGS effects on the drops is here neglected, in order to investigate solely the SGS-flux models and the method for reducing the number of computational drops. The FST models without direct SGS effects on drops were found in Part 1 to be almost as good as those that accounted for such effects deterministically, and superior to those that accounted for them statistically.

This paper is organized as follows: The LES equations are presented in $\S 2$, and models for unclosed terms appearing in these equations, namely the SGS fluxes and the FSTs, are listed. The general approach is to keep the LES methodology as close to the DNS as possible, in order to minimize sources of discrepancy not directly related to explicit modelling issues. The LES initial conditions and numerical procedures are described in $\S 3$, followed in $\S 4$ by an a priori analysis of the interpolation error that arises when the flow field encountered by the drops is represented on the coarser LES grid. LES results are presented in $\S 5$ for a single-phase mixing layer and in $\S 6$ for a two-phase mixing layer laden with evaporating drops. Finally, conclusions and further discussions are presented in $\S 7$.

\section{LES equations and models}

In Part 1, we derived the LES equations appropriate for a flow laden with evaporating drops, with the gas phase formulated in an Eulerian frame and the drops (liquid phase) tracked in a Lagrangian frame. Drops tracked in LES follow the DNS evolution equations; however, in LES one may use computational drops rather than physical drops. Following the SP protocol, the LES equations are derived by spatially filtering the gas-phase DNS equations, and then making various simplifying assumptions which were validated on the DNS database. This procedure is described in detail in Part 1 and the resulting equations are summarized here. The DNS 
equations, also presented in detail in Part 1, are based on the formulation of Miller \& Bellan (1999).

The gas-phase equations are formulated for two species, namely the carrier gas and the vapour evolving from the drops. The coupling describing the interaction between the two phases is provided in the gas-phase conservation equations by source terms and in the drop-evolution equations by the far-field condition for each drop, which is calculated by interpolating the gas-phase solution from the Eulerian grid to the drop location. The drops are treated as point sources of mass, momentum and energy for the gas phase; this treatment is justified by the dilute (i.e. volumetrically small, $O\left(10^{-3}\right)$ ) loading and by the size of each particle being much smaller than the Kolmogorov scale. Each drop is assumed to be spherical, and, consistent with the drop description as a point source, its internal temperature and density are assumed uniform. Furthermore, we neglect unsteady drag and added mass effects, as well as Basset history forces, all of which are small for liquid-to-gas density ratios (Boivin et al. 1998). Drop collisions are assumed negligible.

For ease of notation, we define the vector of gas-phase conservative variables $\phi=\left\{\rho, \rho u_{i}, \rho e_{t}, \rho Y_{V}\right\}$ and denote the flow field as $\phi$, where $\rho$ is the density, $u_{i}$ is the velocity in the $x_{i}$ coordinate direction, $e_{t}$ is the total energy and $Y_{V}$ is the vapour (subscript $V$ ) mass fraction (the carrier gas, subscript $C$, mass fraction is $Y_{C}$; $Y_{C}+Y_{V}=1$ ). For a variable $\varphi, \bar{\varphi}$ denotes spatial filtering whereas $\widetilde{\varphi}=\overline{\rho \varphi} / \bar{\rho}$ denotes the Favre (density-weighted) spatial filtering. We define $Z=\left\{X_{i}, v_{i}, T_{d}, m_{d}\right\}$ as the physical drop field with position $X_{i}$, velocity $v_{i}$, temperature $T_{d}$, and mass $m_{d}$. The drop evolution depends on the gas-phase primitive variables, $\psi(\phi)=\left\{u_{i}, T, Y_{V}, p\right\}$, evaluated either at the drop surface (subscript $s$ ) or at the drop far-field (subscript $f$ ); $T$ is the temperature and $p$ is the pressure. The LES (i.e. filtered) flow field is denoted as $\bar{\phi} ; \bar{Z}$ is the LES (i.e. computational) drop field.

\subsection{Liquid-phase LES equations}

The evolution equations for the computational drops, in a Lagrangian frame, are:

$$
\begin{gathered}
\frac{\mathrm{d} X_{i}}{\mathrm{~d} t}(\bar{Z})=v_{i}, \\
\frac{\mathrm{d} v_{i}}{\mathrm{~d} t}\left(\psi_{f}, \bar{Z}\right)=\frac{1}{m_{d}} F_{i}\left(\psi_{f}, \bar{Z}\right), \\
\frac{\mathrm{d} T_{d}}{\mathrm{~d} t}\left(\psi_{f}, \psi_{s}, \bar{Z}\right)=\frac{1}{m_{d} C_{L}}\left[Q\left(\psi_{f}, \bar{Z}\right)+\dot{m}_{d}\left(\psi_{f}, \psi_{s}, \bar{Z}\right) L_{V}(\bar{Z})\right], \\
\frac{\mathrm{d} m_{d}}{\mathrm{~d} t}\left(\psi_{f}, \psi_{s}, \bar{Z}\right)=\dot{m}_{d}\left(\psi_{f}, \psi_{s}, \bar{Z}\right),
\end{gathered}
$$

where $F_{i}$ is the drag force, $Q$ is the heat flux, $\dot{m}_{d}$ is the evaporation rate, and $C_{L}$ is the heat capacity of the drop liquid. $L_{V}$ is the latent heat of vaporization, which, for the present calorically perfect gas, is a linear function of temperature, $L_{V}=h_{V}^{0}-\left(C_{L}-C_{p, V}\right) T_{d}$, where $h_{V}^{0}$ accounts for the enthalpy difference between the vapour and carrier gas at reference conditions and $C_{p}$ is the gas-phase heat capacity at constant pressure. The detailed expressions for $F_{i}, Q$, and $\dot{m}_{d}$ involve validated correlations for point drops which are based on Stokes drag, with the particle time constant defined by Crowe, Chung \& Troutt (1998) as $\tau_{d}=\rho_{L} d^{2} /(18 \mu)$, where $\rho_{L}$ is the density of the liquid, $d$ is the drop diameter $\left(m_{d}=\rho_{L} \pi d^{3} / 6\right)$ and $\mu$ is the gas-phase 
viscosity:

$$
\begin{aligned}
F_{i}\left(\psi_{f}, \bar{Z}\right) & =\frac{m_{d}}{\tau_{d}} f_{1}\left(u_{i, f}-v_{i}\right), \\
Q\left(\psi_{f}, \bar{Z}\right) & =\frac{m_{d}}{\tau_{d}} \frac{N u}{3 P r} C_{p, f} f_{2}\left(T_{f}-T_{d}\right), \\
\dot{m}_{d}\left(\psi_{f}, \psi_{s}, \bar{Z}\right) & =-\frac{m_{d}}{\tau_{d}} \frac{S h}{3 S c} \ln \left(1+B_{M}\right) .
\end{aligned}
$$

In (2.5), $f_{1}$ is an empirical correlation to correct the Stokes drag for finite drop Reynolds numbers

$$
\begin{gathered}
f_{1}=\frac{1+0.0545 R e_{s l}+0.1 R e_{s l}^{1 / 2}\left(1-0.03 R e_{s l}\right)}{1+a\left|R e_{b}\right|^{b}}, \\
a=0.09+0.077 \exp \left(-0.4 R e_{s l}\right), \quad b=0.4+0.77 \exp \left(-0.04 R e_{s l}\right),
\end{gathered}
$$

based on the slip Reynolds number $R e_{s l}=\left|u_{i, f}-v_{i}\right| \rho d / \mu$, where $\left(u_{i, f}-v_{i}\right)$ is the slip velocity, and on the blowing Reynolds number $\operatorname{Re}_{b}=U_{b} \rho d / \mu$, where $U_{b}$ is the blowing velocity obtained from the mass conservation relation at the drop surface, $\dot{m}_{d}=-\pi \rho d^{2} U_{b}$. The correlation of (2.8) is valid for the ranges $0 \leqslant R e_{s l} \leqslant 100$ and $0 \leqslant R e_{b} \leqslant 10$. In (2.6) and (2.7), the gas-phase Prandtl and Schmidt numbers, $\operatorname{Pr}=\mu C_{p} / \lambda$ and $S c=\mu /(\rho D)$, based on the gas-phase diffusion coefficient $(D)$ and thermal conductivity $(\lambda)$, are assumed constant. In (2.6), $f_{2}=\beta /\left(\mathrm{e}^{\beta}-1\right)$, where $\beta=-1.5 \operatorname{Pr}_{d} \tau_{d} / m_{d}$ is constant for drops obeying the classical ' $d^{2}$ law' (Williams 1965). In (2.7), the mass transfer number is $B_{M}=\left(Y_{V, s}-Y_{V, f}\right) /\left(1-Y_{V, s}\right)$ for which $Y_{V, s}$ is calculated by equating the vapour and liquid fugacities at the surface, i.e. $\left(m_{s} Y_{V, s} / m_{V}\right) p_{s}=p_{\text {sat }}$ where $m=\left(Y_{V} / m_{V}+Y_{C} / m_{C}\right)^{-1}$ is the gas-phase molar mass and $m_{C}$ and $m_{V}$ are the molar masses of the carrier gas and vapour respectively (also $p_{s}=p_{f}$ ). The saturation pressure, $p_{\text {sat }}$, is provided by the Clausius-Clapeyron relation as

$$
p_{s a t}=p_{a t m} \exp \left[\frac{L_{V} m_{V}}{R_{u}}\left(\frac{1}{T_{B, L}}-\frac{1}{T_{d}}\right)\right]
$$

with $p_{a t m}=1 \mathrm{~atm} ; T_{B, L}$ is the liquid saturation temperature at $p_{a t m}$ (i.e. the normal boiling temperature) and $R_{u}$ is the universal gas constant. Finally, the Nusselt, $N u$, and Sherwood, Sh, numbers appearing in (2.6) and (2.7) are empirically modified for convective corrections to heat and mass transfer based on the Ranz-Marshall correlations

$$
N u=2+0.552 R e_{s l}^{1 / 2}(P r)^{1 / 3}, \quad S h=2+0.552 R e_{s l}^{1 / 2}(S c)^{1 / 3} .
$$

Except for $\tau_{d}$, which depends on $\mu,(2.5)-(2.7)$ depend essentially on ratios of transport properties through non-dimensional numbers. Therefore, the value of $\tau_{d}$ and thus, for a given liquid and drop size, the value of $\mu$ determines the interaction time between drops and gas.

These expressions for $F_{i}, Q$, and $\dot{m}_{d}$ are such that the drop evolution equations are highly nonlinear functions of both the drop state and the gas phase. The unfiltered flow field $(\psi)$ must be calculated from $\bar{\phi}$ using a model, as discussed in $\S 2.3$. 


\subsection{Gas-phase LES equations}

The adopted form of the gas-phase LES equations, resulting from the analysis in Part 1, is:

$$
\begin{gathered}
\frac{\partial \bar{\rho}}{\partial t}+\frac{\partial\left(\bar{\rho} \tilde{u}_{j}\right)}{\partial x_{j}}=\bar{S}_{I}, \\
\frac{\partial\left(\bar{\rho} \tilde{u}_{i}\right)}{\partial t}+\frac{\partial\left(\bar{\rho} \tilde{u}_{i} \tilde{u}_{j}\right)}{\partial x_{j}}=-\frac{\partial[p(\bar{\phi})]}{\partial x_{i}}+\frac{\partial \sigma_{i j}(\bar{\phi})}{\partial x_{j}}+\bar{S}_{I I, i}-\frac{\partial\left(\bar{\rho} \tau_{i j}\right)}{\partial x_{j}}, \\
\frac{\partial\left(\bar{\rho} \tilde{e}_{t}\right)}{\partial t}+\frac{\partial\left(\bar{\rho} \tilde{e}_{t} \tilde{u}_{j}\right)}{\partial x_{j}}=-\frac{\partial\left[p(\bar{\phi}) \tilde{u}_{j}\right]}{\partial x_{j}}-\frac{\partial q_{j}(\bar{\phi})}{\partial x_{j}}+\frac{\partial\left[\sigma_{i j}(\bar{\phi}) \tilde{u}_{i}\right]}{\partial x_{j}}+\bar{S}_{I I I}-\frac{\partial \bar{\rho} \zeta_{j}}{\partial x_{j}}-\frac{\partial}{\partial x_{j}}\left[\bar{\rho} \tau_{i j} \tilde{u}_{i}\right], \\
\frac{\partial\left(\bar{\rho} \tilde{Y}_{V}\right)}{\partial t}+\frac{\partial\left(\bar{\rho} \tilde{Y}_{V} \tilde{u}_{j}\right)}{\partial x_{j}}=-\frac{\partial j_{V j}(\bar{\phi})}{\partial x_{j}}+\bar{S}_{I}-\frac{\partial\left(\bar{\rho} \eta_{j}\right)}{\partial x_{j}},
\end{gathered}
$$

where the SGS fluxes are

$$
\tau_{i j}=\widetilde{u_{i} u_{j}}-\tilde{u}_{i} \tilde{u}_{j}, \quad \zeta_{j}=\widetilde{h u_{j}}-\tilde{h} \tilde{u}_{j}, \quad \eta_{j}=\widetilde{Y_{V} u_{j}}-\tilde{Y}_{V} \tilde{u}_{j} .
$$

The internal energy $\left(e=e_{t}-u_{i} u_{i} / 2\right)$, the enthalpy $(h=e+p / \rho), p$ and $T$ have the same form as for DNS, for a calorically perfect gas:

$$
\begin{gathered}
p(\phi)=\rho R(\phi) T(\phi), \\
h(\phi)=C_{p}(\phi) T(\phi)+h_{V}^{0} Y_{V}=h_{C} Y_{C}+h_{V} Y_{V},
\end{gathered}
$$

where $R(\phi)=R_{u} / m(\phi) ; C_{p}(\phi)=C_{p, V} Y_{V}+C_{p, C} Y_{C}$ with $C_{p, C}$ and $C_{p, V}$ here assumed constant. The viscous stress $\left(\sigma_{i j}\right)$, the heat flux $\left(q_{j}\right)$, and the vapour mass flux $\left(j_{V j}\right)$ also have the same form as for DNS:

$$
\begin{gathered}
\sigma_{i j}(\phi)=2 \mu\left(S_{i j}-\frac{1}{3} S_{k k} \delta_{i j}\right), \quad S_{i j}(\phi)=\frac{1}{2}\left(\frac{\partial u_{i}}{\partial x_{j}}+\frac{\partial u_{j}}{\partial x_{i}}\right), \\
j_{V j}(\phi)=-\rho D\left[\frac{\partial Y_{V}}{\partial x_{j}}+\frac{\left(m_{C}-m_{V}\right)}{m(\phi)} \frac{Y_{V} Y_{C}}{p(\phi)} \frac{\partial[p(\phi)]}{\partial x_{j}}\right], \\
q_{j}(\phi)=-\lambda \frac{\partial[T(\phi)]}{\partial x_{j}}+\left[h_{V}(\phi)-h_{C}(\phi)\right] j_{V j}(\phi),
\end{gathered}
$$

where $S_{i j}$ is the rate of strain and thermal diffusion effects have been neglected. In (2.19)-(2.21), $D, \lambda$ and $\mu$ are assumed constant.

Equations (2.12)-(2.15) assume that

$$
\frac{1}{2}\left(\overline{\rho u_{i} u_{i} u_{j}}-\overline{\rho u_{i} u_{i}} \tilde{u}_{j}\right)=\bar{\rho} \tau_{i j} \tilde{u}_{i},
$$

and that $\overline{f(\phi)}=f(\bar{\phi})$, i.e.

$$
\begin{array}{cll}
\tilde{e}=e(\bar{\phi}), & \bar{T}=T(\bar{\phi}), \quad \tilde{T}=T(\bar{\phi}), \quad \bar{p}=p(\bar{\phi}), \quad \tilde{h}=h(\bar{\phi}), \\
\bar{\sigma}_{i j}=\sigma_{i j}(\bar{\phi}), \quad \overline{u_{i} \sigma_{i j}}=\tilde{u}_{i} \sigma_{i j}(\bar{\phi}), \quad \bar{j}_{V j}=j_{V j}(\bar{\phi}), \quad \bar{q}_{j}=q_{j}(\bar{\phi}) .
\end{array}
$$

These assumptions were assessed on the DNS database and found to be valid (Part 1). Equations (2.12)-(2.15) for $\bar{\phi}$ contain terms that cannot be computed directly from $\bar{\phi}$ and that therefore must be modelled, namely (i) the FSTs $\left(\bar{S}=\left\{\bar{S}_{I}, \bar{S}_{I I, i}, \bar{S}_{I I I}, \bar{S}_{I}\right\}\right)$ and (ii) the SGS fluxes $\left(\tau_{i j}, \zeta_{j}, \eta_{j}\right)$. 


\subsection{Models for filtered source terms}

For the FSTs, $\bar{S}$ is properly interpreted by considering a physical drop $\alpha$ located at $\boldsymbol{X}_{\alpha}$ within the filtering volume $V_{f}$ and its contribution within that volume:

$$
\bar{S}_{\alpha}(\boldsymbol{x})=\int_{V_{f}}\left[S_{d}\right]_{\alpha} \delta\left(\boldsymbol{y}-\boldsymbol{X}_{\alpha}\right) G(\boldsymbol{x}-\boldsymbol{y}) \mathrm{d} \boldsymbol{y},
$$

where the delta function $\delta\left(\boldsymbol{y}-\boldsymbol{X}_{\alpha}\right)$ restricts the action of the drop source term contribution $S_{d}$ to the drop location $\boldsymbol{X}_{\alpha}$. When the filter function $G$ is a top-hat filter, the exact FSTs are

$$
\bar{S}\left(\psi_{f}, Z\right)=\sum_{\alpha=1}^{N_{\alpha}} \bar{S}_{\alpha}=\frac{1}{V_{f}} \sum_{\alpha=1}^{N_{\alpha}}\left[S_{d}\left(\psi_{f}, Z\right)\right]_{\alpha},
$$

a volume-average over the $N_{\alpha}$ physical drops within $V_{f}$, with

$$
\begin{aligned}
S_{d}\left(\psi_{f}, Z\right) & =\left\{S_{I, d}, S_{I I, i, d}, S_{I I I, d}, S_{I, d}\right\}, \\
S_{I, d} & =-\dot{m}_{d}, \\
S_{I I, i, d} & =-\left[F_{i}+\dot{m}_{d} v_{i}\right], \\
S_{I I I, d} & =-\left[F_{i} v_{i}+Q+\dot{m}_{d}\left(\frac{1}{2} v_{i} v_{i}+C_{p, V} T_{d}+h_{V}^{0}\right)\right] .
\end{aligned}
$$

In LES, neither $Z$ nor $\psi$ is available, therefore the FSTs must be modelled from $\bar{Z}$ and $\bar{\phi}$; these modelling requirements are specific to TP flows. In Part 1, we proposed several models for the FSTs and assessed them a priori. For $\bar{Z}$, we proposed to allow each computational drop to replace $N_{R}$ physical drops and to compute the contribution of each computational drop as in the DNS. The resulting model for $\bar{S}$ was

$$
\bar{S}_{m}\left(\psi_{f, m}(\bar{\phi}), \bar{Z}\right)=N_{R} \sum_{\beta=1}^{N_{\beta}} \frac{1}{V_{f}}\left[S_{d}\left(\psi_{f, m}(\bar{\phi}), \bar{Z}\left(N_{R}\right)\right)\right]_{\beta},
$$

where the summation is over the $N_{\beta}$ computational drops within $V_{f}$, and $S_{d}$ has the functional form of (2.27) with $\psi_{f, m}$ denoting the model for $\psi_{f}$. In (2.31), the representative nature of each drop is entirely embodied in the parameter $N_{R}$, which is constant.

For rendering $\psi$, we assessed the filtered flow field ('baseline' model) as well as a 'random' model and a more sophisticated 'deterministic' model, the latter two using the SGS variance $\sigma_{\mathrm{SGS}}^{2}(\psi)=\widetilde{\psi^{2}}-(\tilde{\psi})^{2}$, which we determined could be modelled in a similar manner as the SGS fluxes. The assessment of Part 1 was that, for the purpose of calculating drop source terms, the filtered flow field performed better than the random model but not as well as the deterministic model of the unfiltered variables. However, a complex FST-model error behaviour that was dependent on both the filter width and on $N_{R}$, as well as the inability of an a priori study to predict the effect of the FSTs on the flow evolution, showed the need to perform a more cogent assessment of these models by means of a posteriori studies. In the present study, we use the filtered flow field in place of the unfiltered flow field in calculating the source terms. This decision is motivated by the increased complexity of the random and deterministic models due to the need to model $\sigma_{\mathrm{SGS}}$; future studies may examine whether the additional complexity of these models leads to improved accuracy in LES. Using the filtered flow field to represent the unfiltered one amounts to neglecting direct SGS effects on drop evolution (as done by Boivin et al. 2000 and Yamamoto et al. 2001). 
Therefore, the FST model for the present study is $(2.31)$ with $\psi_{f, m}=\tilde{\psi}_{f}$ :

$$
\bar{S}\left(\tilde{\psi}_{f}, \bar{Z}\right)=N_{R} \sum_{\beta=1}^{N_{\beta}} \frac{1}{V_{f}}\left[S_{d}\left(\tilde{\psi}_{f}, \bar{Z}\left(N_{R}\right)\right)\right]_{\beta},
$$

and the only parameter is $N_{R}$. The selection of $N_{R}$ involves an appraisal of the possible increase in computational efficiency, weighed against a likely loss of accuracy, as $N_{R}$ is increased. Consistent with this FST model, the computational-drop evolution is given by (2.1)-(2.4), with $\psi$ replaced by $\tilde{\psi}$.

\subsection{Subgrid-scale-flux models}

For compactness of notation, we denote the SGS fluxes for a variable $\varphi$ as

$$
\theta_{j}(\varphi)=\left(\widetilde{\varphi u_{j}}-\tilde{\varphi} \tilde{u}_{j}\right)
$$

where $\theta_{j}\left(u_{i}\right)=\tau_{i j}, \theta_{j}\left(Y_{V}\right)=\eta_{j}$ and $\theta_{j}(h)=\zeta_{j}$. The model for $\theta_{j}(\varphi)$, to be calculated on the filtered flow field $(\bar{\phi})$ is denoted $\mu_{j}(\tilde{\varphi} ; \bar{\phi}, \bar{\Delta}$ ) (associated with the filter width $\bar{\Delta}$ and with the velocity $\left.\tilde{u}_{j}\right) ; \mu_{j}$ does not contain the model coefficient. We define a generic model coefficient $\chi$,

$$
\theta_{j}(\varphi)=\chi(\tilde{\varphi} ; \bar{\phi}, \bar{\Delta}) \mu_{j}(\tilde{\varphi} ; \bar{\phi}, \bar{\Delta})
$$

In the most general case, $\chi$ is spatially and temporally varying, and moreover has different values for each SGS flux to be modelled. Our LES will consider two more restrictive cases where these $\chi$ have the same value for all (or a subset of) SGS fluxes and (i) are temporally and spatially constant and must be externally specified (in our case through calibration on a DNS database), or (ii) are temporally varying but spatially constant in the domain (or on homogeneous planes) and are dynamically computed as part of the LES solution.

For the SGS fluxes defined in (2.16), we consider here the following three typical SGS models:

(a) The Smagorinsky (SM) model is (see Smagorinksy 1993)

$$
\begin{aligned}
\varphi=u_{i}: \mu_{j}(\tilde{\varphi} ; \bar{\phi}, \bar{\Delta}) & =\mu_{j}^{0}\left(\tilde{u}_{i} ; \bar{\phi}, \bar{\Delta}\right)+\left(\chi_{\text {trace }} \delta_{i j} / 3\right) \mu_{k}\left(\tilde{u}_{k} ; \bar{\phi}, \bar{\Delta}\right), \\
\mu_{j}^{0}\left(\tilde{u}_{i}\right) & =-\bar{\Delta}^{2} S(\bar{\phi}) S_{i j}^{0}(\bar{\phi}), \\
\varphi=Y_{V}, h: \mu_{j}(\tilde{\varphi} ; \bar{\phi}, \bar{\Delta}) & =-\bar{\Delta}^{2} S(\bar{\phi}) \frac{1}{2} \frac{\partial \tilde{\varphi}}{\partial x_{j}},
\end{aligned}
$$

where $\chi \mu_{j}^{0}\left(\tilde{u}_{i}\right)$ is the model for $\tau_{i j}^{0}, \chi \chi_{\text {trace }} \mu_{k}\left(\tilde{u}_{k}\right)$ is the model for $\tau_{k k}$, the superscript zero denotes a traceless tensor (e.g. $\left.\tau_{i j}^{0}=\tau_{i j}-\left(\tau_{k k} / 3\right) \delta_{i j}, \tau_{k k}^{0}=0\right)$ and

$$
S^{2}(\phi)=S_{i j}(\phi) S_{i j}(\phi)
$$

The consistency conditions on $\tau_{i j}$ are most simply satisfied by having $\chi$ and $\chi_{\text {trace }}$ take on the same values for all $\mu_{j}\left(u_{i}\right)$. To model $\tau_{k k}$, which may be important for compressible turbulence (Erlebacher et al. 1992), we use the Yoshizawa (1986) (YO) model:

$$
\mu_{k}\left(\tilde{u}_{k} ; \bar{\phi}, \bar{\Delta}\right)=\bar{\Delta}^{2} S^{2}(\bar{\phi}) .
$$

(b) The gradient (GR) model is (Clark, Ferziger \& Reynolds 1979)

$$
\varphi=u_{i}, Y_{V}, h: \mu_{j}(\tilde{\varphi} ; \bar{\phi}, \bar{\Delta})=\bar{\Delta}^{2} \frac{\partial \tilde{\varphi}}{\partial x_{k}} \frac{\partial \tilde{u}_{j}}{\partial x_{k}} .
$$


(c) The scale-similarity (SS) model is (Bardina, Ferziger \& Reynolds 1980)

$$
\varphi=u_{i}, Y_{V}, h: \mu_{j}(\tilde{\varphi} ; \bar{\phi}, \bar{\Delta})=\left(\widehat{\tilde{\varphi} \tilde{u}_{j}}-\widehat{\tilde{\varphi}} \widehat{\tilde{u}}_{j}\right),
$$

where the overhat $\left({ }^{\wedge}\right.$ ) denotes (unweighted) filtering at the test-filter level $\hat{\Delta} \geqslant \bar{\Delta}$.

\subsubsection{Constant-coefficient models}

For the constant-coefficient versions of the Smagorinsky, gradient and scalesimilarity models, denoted SMC, GRC and SSC, all SGS fluxes use the same coefficient value, that is, in the notation of Part 1: $\chi=C_{\mathrm{SM}}, \chi \chi_{\text {trace }}=C_{\mathrm{YO}}(\mathrm{SMC})$; $\chi=C_{\mathrm{GR}}(\mathrm{GRC}) ; \chi=C_{\mathrm{SS}}(\mathrm{SSC})$. The constant coefficient must be externally specified, either from theoretical considerations, or from calibration with DNS or experiments. For the GR model, theoretically (i.e. from a Taylor series expansion, see Appendix A of Part 1) $C_{\mathrm{GR}}=1 / 12$ for a cubic top-hat filter. Scale-similarity implies that $C_{\mathrm{SS}}=1$ for identical grid and test filters (Bardina et al. 1980; Pruett \& Adams 2000). However, practically, its true value depends on the situation under study as well as on the filter type and on the ratio $\hat{\Delta} / \bar{\Delta}$ (see Appendix B of Part 1).

One may also construct more general SGS-flux models having a different coefficient for each type of flux; for example, a four-coefficient model

$$
\chi\left(\tau_{i j, i=j}\right)=C_{\tau d}, \quad \chi\left(\tau_{i j, i \neq j}\right)=C_{\tau x}, \quad \chi\left(\zeta_{j}\right)=C_{\zeta}, \quad \chi\left(\eta_{j}\right)=C_{\eta},
$$

and three-coefficient models

$$
\begin{gathered}
\chi\left(\tau_{i j}\right)=C_{\tau}, \quad \chi\left(\zeta_{j}\right)=C_{\zeta}, \quad \chi\left(\eta_{j}\right)=C_{\eta} ; \\
\chi\left(\tau_{i j, i=j}\right)=C_{\tau d}, \quad \chi\left(\tau_{i j, i \neq j}\right)=C_{\tau x}, \quad \chi\left(\zeta_{j}\right)=\chi\left(\eta_{j}\right)=C_{\zeta \eta} .
\end{gathered}
$$

\subsubsection{Dynamic models}

A more sophisticated but more computationally expensive approach than utilizing constant coefficients is to determine the model coefficients from the LES solution through dynamic modelling. Basically, dynamic modelling is an application of the SS concept, in that it attempts to deduce the SGS behaviour from that of the small resolved scales (SRSs). The essence of the method is to relate the grid-level SGS flux, $\theta_{j}$, and the test-level SGS flux,

$$
T_{j}(\varphi)=\left(\widehat{\widehat{\varphi u_{j}}}-\widehat{\widetilde{\varphi}} \widehat{\tilde{u}}_{j}\right),
$$

to the test-level resolved flux, $L_{j}$, through the Germano identity (Germano et al. 1991):

$$
L_{j}(\varphi) \equiv \widehat{\tilde{\varphi} \tilde{u}_{j}}-\widehat{\widetilde{\varphi}} \widehat{\tilde{u}}_{j}=T_{j}-\hat{\theta}_{j}
$$

Since the grid-level SGS-flux model (without coefficient) has been previously defined as $\mu_{j}(\tilde{\varphi} ; \bar{\phi}, \bar{\Delta})$, the test-level SGS-flux model is $\mu_{j}(\widehat{\hat{\varphi}} ; \widehat{\bar{\phi}}, \widehat{\bar{\Delta}})$, associated with the filter width $\widehat{\bar{\Delta}}$ and with the velocity $\widehat{\widetilde{u}}_{j}$. Here $\widehat{\bar{\Delta}}$ is the effective filter width, not actually used for filtering, that corresponds to filtering at $\bar{\Delta}$ followed by filtering at $\hat{\Delta}$, and whose value depends on the filter type. For the top-hat filter used here, it is optimally approximated by $\widehat{\widehat{\Delta}}^{2}=\bar{\Delta}^{2}+\hat{\Delta}^{2}$ (Vreman, Guerts \& Kuerten 1997); this value is the exact one for a Gaussian filter, whereas $\widehat{\bar{\Delta}}=\hat{\Delta}$ for a spectral cut-off filter. Then $L_{j}$ can be modelled as

$$
M_{j}(\varphi)=\mu_{j}(\widehat{\tilde{\varphi}})-\widehat{\mu_{j}(\tilde{\varphi})} .
$$


The model coefficient can be extracted using a least-squares fit (Lilly 1992),

$$
C_{H}(\varphi)=\frac{\left\langle L_{j}(\varphi) M_{j}(\varphi)\right\rangle}{\left\langle M_{k}(\varphi) M_{k}(\varphi)\right\rangle}, \quad C_{D}(\varphi)=\frac{\left\langle\left\langle L_{j}(\varphi) M_{j}(\varphi)\right\rangle\right\rangle}{\left\langle\left\langle M_{k}(\varphi) M_{k}(\varphi)\right\rangle\right\rangle},
$$

where, for the present mixing layer configuration, \langle\rangle denotes averaging over homogeneous $\left(x_{1}, x_{3}\right)$-planes while $\langle\langle\rangle\rangle$ denotes averaging over the entire domain. Using the multi-coefficient formulation (2.42), the summation over repeated indices would be over three quantities for the SGS-stress diagonal terms, over three quantities for the SGS-stress off-diagonal terms, over three quantities for the SGS species flux and over three quantities for the SGS enthalpy flux. (The use of dimensional variables in the present formulation necessitates the separate computation of the coefficient for each type of SGS flux.) The dynamic coefficients to replace the constant coefficients are computed using:

(a) for the dynamic Smagorinsky (SMD) model, $\mu_{j}$ from (2.35)-(2.39);

(b) for the dynamic gradient (GRD) model, $\mu_{j}$ from (2.40).

Further details on the implementation of the dynamic models are provided in $\$ 3.2 .2$ below. In view of the observation in Part 1 that the scale-similarity and gradient models have much better correlations with the SGS fluxes than does the Smagorinsky model, we do not consider 'mixed' models, in which the (dynamic) Smagorinsky model is used to add dissipation to the other two models (thus our dynamic gradient model differs from that of Vreman et al. 1997).

\section{LES methodology}

The LES results are to be compared to the filtered-and-coarsened (FC) DNS, filtered at the desired width $\bar{\Delta}$ and then coarsened so that only the DNS grid points corresponding to the LES grid are used. For TP cases, FC-DNS also means that a subset of the DNS physical drops are extracted, to create a reduced drop field using the same $N_{R}$ as the LES. Although the goal of TP LES is to reproduce the physical flow and drop fields (represented here by DNS), the formal comparison of the LES should be made to FC-DNS, which represents the best possible result for the flow-field resolution and the number of computational drops selected for the LES. The determination of whether the LES resolution is adequate for the physical problem should be considered separately using grid-resolution studies for the flow field and/or different $N_{R}$ values for the drop field; in the general situation where direct comparison with the physical problem is not possible, the LES with different grids and/or $N_{R}$ values might be compared to one another to obtain a grid-and/or $N_{R}$-converged solution.

The LES initial condition is the FC-DNS initial condition. Generally, when an initial DNS flow field is unavailable, an initial LES condition has to be carefully calculated on a coarser grid in order to avoid poor accuracy or numerical instabilities. The LES are performed using the same numerical scheme as the DNS, namely fourthorder explicit time integration and eighth-order central finite-differences (Kennedy \& Carpenter 1994) with fourth-order interpolation of gas-phase variables from grid points to drop locations. The flow configuration and initial conditions are summarized below; further details can be found in Part 1.

\subsection{Flow configuration and initial conditions}

The mixing-layer geometric configuration is illustrated in figure 1, where the streamwise $\left(x_{1}\right)$, the cross-stream $\left(x_{2}\right)$, and the spanwise $\left(x_{3}\right)$ coordinates are shown, and 


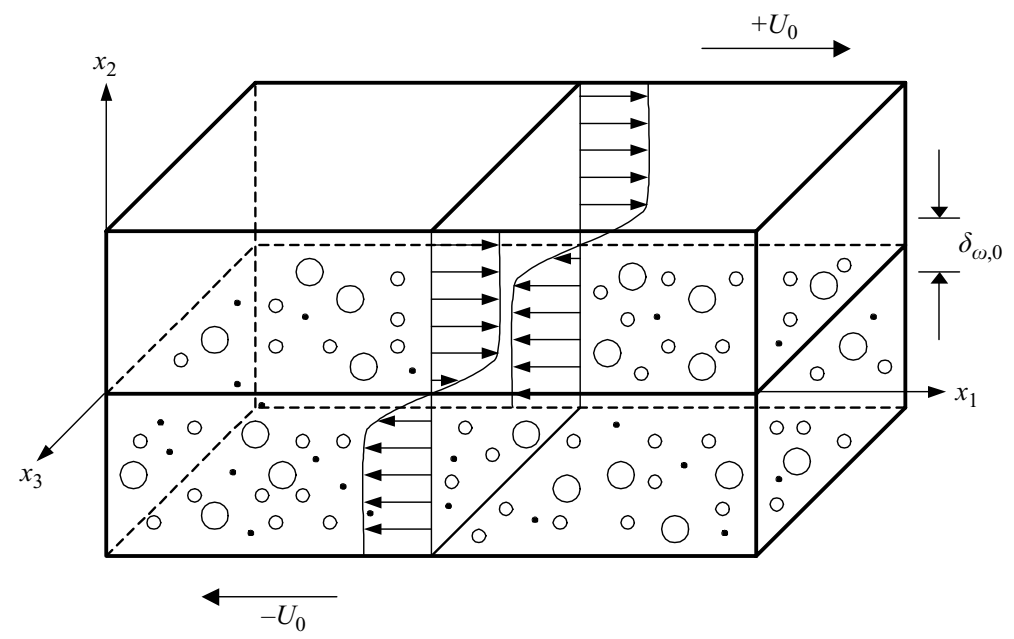

FIGURE 1. Mixing-layer configuration.

the respective domain lengths are $L_{1}, L_{2}$ and $L_{3}$. Periodic boundary conditions are used in the $x_{1}$ - and $x_{3}$-directions, and adiabatic slip wall conditions (see Poinsot \& Lele 1992) are employed for the $x_{2}$ boundaries. Initially, the gas phase consists only of the carrier gas (no vapour). To promote layer growth, the layer is initially perturbed so as to induce roll-up and pairing. The evolution of the layer comprises two pairings of the four initial spanwise vortices to form a single vortex. The initial (subscript 0) free-stream velocity, $U_{0}=M_{c, 0} a_{C, 0}$, is calculated from a specified value of the convective Mach number $\left(M_{c, 0}\right)$ based on the carrier gas initial speed of sound $\left(a_{C, 0}\right)$. The initial vorticity thickness is $\delta_{\omega, 0}=\delta_{\omega}(0)$ where $\delta_{\omega}(t)=\Delta U_{0} /$ $\left(\partial\left\langle u_{1}\right\rangle / \partial x_{2}\right)_{\max }$, with $\Delta U_{0}=2 U_{0}$ being the velocity difference across the layer; initially, $\left\langle u_{1}\right\rangle$ has an error-function profile. The specified value of the initial Reynolds number, $R e_{0}=\rho_{0} \Delta U_{0} \delta_{\omega, 0} / \mu$, where $\rho_{0}$ is the initial gas density, is used to calculate $\mu$. The thermal conductivity and diffusivity are then computed using this value of $\mu$ and (constant) specified values of Prandtl and Schmidt numbers ( $\operatorname{Pr}=S c=0.67)$. All thermophysical properties are the same as those employed in the simulations of Miller \& Bellan (2000) using air as the carrier gas and decane as the drop liquid. For TP flow, the drops are initially distributed randomly throughout the $x_{2}<0$ domain. All the drops have the same initial temperature, $T_{d, 0}$, set lower than the initial carriergas temperature, $T_{C, 0}$, to promote evaporation. Initially the drops have the same velocity as the gas phase at their location. The initial drop size is specified through the initial Stokes number $S t_{0}$ where $S t=\tau_{d} \Delta U_{0} / \delta_{\omega, 0}$. The initial number of physical drops $N_{d, 0}$ is determined by the initial mass loading $M L_{0}$ (initial ratio of liquid mass to mass of carrier gas in the drop-laden part of the domain). As drops heat up and evaporate, their size decreases; drops smaller than a certain mass (corresponding to a Stokes number of 0.1 ) are removed from the domain because of their negligible dynamic and thermodynamic impact on the flow.

The DNS cases considered here are listed in table 1 . All cases have $R e_{0}=600$, which is the higher Reynolds number simulated in Part 1. These cases comprise SP600 $\left(M L_{0}=0\right)$ and TP600a2 $\left(M L_{0}=0.2\right)$, which are the two simulations that were analysed on an a priori basis in Part 1 , and TP600a5 $\left(M L_{0}=0.5\right)$ for which application of our models represents an additional test; that is, we study the applicability of 


\begin{tabular}{lcccccccccc}
\hline \multicolumn{1}{c}{ Run } & $R e_{0}$ & $M L_{0}$ & $N_{d, 0}$ & $t_{p 1}^{*}$ & $t_{p 2}^{*}$ & $t_{\text {peak }}^{*}$ & $t_{\text {trans }}^{*}$ & $\begin{array}{c}\delta_{m} / \delta_{\omega, 0} \\
\text { at } t_{\text {trans }}^{*}\end{array}$ & $\begin{array}{c}R e_{m} \\
\text { at } t_{\text {trans }}^{*}\end{array}$ & $\begin{array}{c}\text { CPU-hours } \\
\text { (estimated) }\end{array}$ \\
SP600 & 600 & 0 & 0 & 45 & 75 & 100 & 100 & 2.485 & 1491 & 1054 \\
TP600a2 & 600 & 0.2 & 2993630 & 45 & 80 & 105 & 105 & 2.627 & 1576 & 2252 \\
TP600a5 & 600 & 0.5 & 7484075 & 45 & 80 & 105 & 105 & 2.613 & 1568 & 2981
\end{tabular}

$t_{p 1}^{*}$ and $t_{p 2}^{*}$ : first and second pairing times; $t_{\text {peak }}^{*}$ : momentum-thickness peak after second pairing; $t_{\text {trans }}^{*}$ : transition time; $\left(t_{p 1}^{*}, t_{p 2}^{*}, t_{\text {peak }}^{*}\right.$ and $t_{\text {trans }}^{*}$ are rounded to the nearest $t^{*} \equiv t \Delta U_{0} / \delta_{\omega, 0}$ divisible by 5$)$.

For all cases: $M_{c, 0}=0.35, T_{C, 0}=375 \mathrm{~K}, \rho_{0}=0.9415 \mathrm{~kg} \mathrm{~m}^{-3}, \Delta U_{0}=271.69 \mathrm{~m} \mathrm{~s}^{-1}, \delta_{\omega, 0}=6.859 \times$ $10^{-3} \mathrm{~m}, L_{1}=0.2 \mathrm{~m}, L_{2}=0.22 \mathrm{~m}, L_{3}=0.12 \mathrm{~m}, N_{1}=288, N_{2}=320, N_{3}=176 . N_{i}$ is the number of grid points in the $x_{i}$ direction.

For drop laden cases: $T_{d, 0}=345 \mathrm{~K}, \rho_{L}=642 \mathrm{~kg} \mathrm{~m}^{-3}, T_{B, L}=447.7 \mathrm{~K}, S t_{0}$ has Gaussian distribution with mean 3 , standard deviation 0.5 .

CPU-hours: aggregate over parallel processors on SGI Origin 2000.

TABLE 1. Summary of DNS database.

models derived from the DNS of SP600 and TP600a2 to LES of these two cases and to LES at a higher value of $M L_{0}$. The layer growth is measured using the momentum thickness, $\delta_{m}$, calculated as

$$
\delta_{m}=\frac{\int_{x_{2, \min }}^{x_{2, \max }}\left[\left\langle\rho u_{1}\right\rangle_{x_{2, \max }}-\left\langle\rho u_{1}\right\rangle\right]\left[\left\langle\rho u_{1}\right\rangle-\left\langle\rho u_{1}\right\rangle_{x_{2, \min }}\right] d x_{2}}{\left(\left\langle\rho u_{1}\right\rangle_{x_{2, \max }}-\left\langle\rho u_{1}\right\rangle_{x_{2, \min }}\right)^{2}}
$$

where $x_{2, \max }=L_{2} / 2$ and $x_{2, \min }=-L_{2} / 2$ are the slip wall coordinates. In the DNS, the time at which transition was attained, $t_{\text {trans }}^{*}$, listed in table 1 , was determined to be the same as $t_{\text {peak }}^{*}$, the time of the peak in $\delta_{m}$ after the second pairing, provided that the layer displayed transitional aspects. In particular, the velocity-fluctuation-based spectra displayed a smooth aspect at that time (see Part 1), which indicated that transition was indeed achieved. Also, the momentum-thickness Reynolds number, $R e_{m}=\rho_{0} \Delta U_{0} \delta_{m} / \mu$, listed in table 1 is about 1500 at $t_{\text {trans }}^{*}$, above that typical of the laminar regime.

We use an LES grid coarser than the DNS grid, with $\Delta x_{\text {LES }}=4 \Delta x_{\text {DNS }}$, where $\Delta x_{\mathrm{DNS}}=\max \left(\Delta x_{1}, \Delta x_{2}, \Delta x_{3}\right)$ on the DNS grid, and set $\bar{\Delta}=2 \Delta x_{\mathrm{LES}}$. This is the larger of the two filter widths $\left(\bar{\Delta}=4 \Delta x_{\mathrm{DNS}}\right.$ and $\left.\bar{\Delta}=8 \Delta x_{\mathrm{DNS}}\right)$ that were analysed in Part 1. The grid coarsening selected was the maximum possible for this grid while ensuring that the high-order (12-point) boundary stencils did not include points from within the mixing region. The choice $\bar{\Delta}=2 \Delta x_{\text {LES }}$ allows us to use the scale-similarity model with $\hat{\Delta}=\bar{\Delta}$, since it permits more than one grid point in the filtering volume. Note that each drop will be in several filtering volumes, since the filtering volumes associated with adjacent grid points will overlap. This is not an inconsistency, as it is accounted for in the rigorously developed methodology, in which each drop's contribution to the filtered flow field is averaged over the filtering volume. Therefore the contributions of a given drop to all the filtering volumes in which it resides are consistently accounted for. The type of LES filter, in this case a cubic top-hat filter, explicitly appears in

(a) computing the initial condition, 


\begin{tabular}{|c|c|c|c|c|c|c|c|c|c|}
\hline \multicolumn{5}{|c|}{ Smagorinsky model } & \multicolumn{5}{|c|}{ Gradient model } \\
\hline & SP600 & TP600a2 & TP600a5 & Ave. & & SP600 & ТР600a2 & TP600a5 & Ave. \\
\hline$C_{\tau}$ & 0.0420 & 0.0430 & 0.0443 & 0.0431 & $C_{\tau d}$ & 0.1690 & 0.1763 & 0.1748 & 0.1734 \\
\hline$C_{\zeta}$ & 00747 & & & & $C_{\tau x}$ & 0.1330 & $\begin{array}{l}0.1353 \\
01329\end{array}$ & 0.1347 & 0.1343 \\
\hline$C_{\eta}$ & $\begin{array}{c}0.0141 \\
-\end{array}$ & $\begin{array}{l}0.1270 \\
0.1274\end{array}$ & 0.1228 & 0.1251 & $\begin{array}{l}c_{\zeta} \\
C_{\eta}\end{array}$ & $\begin{array}{c}0.1339 \\
-\end{array}$ & $\begin{array}{l}0.1329 \\
0.1332\end{array}$ & 0.1312 & $\begin{array}{l}0.1329 \\
0.1322\end{array}$ \\
\hline
\end{tabular}

TABLE 2. Average slopes for multi-coefficient calibration of the constant-coefficient Smagorinsky and gradient models. Slope (exact/model) from least-squares fit of SGS flux to SGS-flux model. Calculations performed on DNS databases at $t_{\text {trans }}^{*}$ listed in table 1 using $\bar{\Delta}=8 \Delta x_{\text {DNS. }}$

(b) computing the SGS quantities (if the SSC or a dynamic SGS model is used), and

(c) computing the FSTs.

\subsection{SGS-flux model implementation}

\subsubsection{Constant-coefficient models}

The constant-coefficient SGS-flux models (SMC, GRC and SSC) were implemented as discussed in $\S 2.4 .1$, using coefficient values of

$$
\left.\begin{array}{l}
C_{\mathrm{SM}}=0.072, \quad C_{\mathrm{YO}}=0.314 ; \quad C_{\mathrm{GR}}=0.152 ; \\
C_{\mathrm{SS}}=1.996 \text { for } \hat{\Delta}=\bar{\Delta}, \quad C_{\mathrm{SS}}=0.808 \text { for } \hat{\Delta}=2 \bar{\Delta} .
\end{array}\right\}
$$

These coefficients were calibrated in Part 1 using transitional states for DNS cases SP600 and TP600a2, and were found to be statistically equivalent for SP600 and TP600a2. Notably, the GRC and SSC models showed excellent correlations (over $90 \%$ ) with the SGS fluxes, while the SMC model had poor correlations (about 30\%).

The multi-coefficient calibration for the Smagorinsky and gradient models is presented in table 2 for $\bar{\Delta}=8 \Delta x_{\mathrm{DNS}}$ (results for $\bar{\Delta}=4 \Delta x_{\mathrm{DNS}}$ were similar), where for the Smagorinsky model we use (2.43) while for the gradient model we use (2.42). (Multi-coefficient models were not considered for the scale-similarity model since the single-coefficient model was robust.) For the Smagorinsky model, $C_{\tau}$ is less than the calibrated single-coefficient value of 0.072 , while the other two coefficients $C_{\zeta}$ and $C_{\eta}$ are greater. For the gradient model, $C_{\tau d}$ is greater than the single coefficient value of 0.152 , while the other three coefficients are smaller.

Although the (single and multiple) constant-coefficient models were adequate for SP600 LES, they were not consistently numerically stable for TP600a2 and TP600a5 LES; therefore only the single-coefficient results will be presented. Furthermore, the SSC model with $\hat{\Delta}=2 \bar{\Delta}$ was numerically unstable, therefore only the SSC model with $\hat{\Delta}=\bar{\Delta}$ was used. (Numerical instability was manifested by the code crashing shortly before the transition time listed in table 1.)

\subsubsection{Dynamic models}

For the dynamic models conceptualized in $\S 2.4 .2$, after some experimentation we settled on a three-coefficient SMD model (2.43) and a four-coefficient GRD model (2.42), with domain averaging for the SGS stress, domain or homogeneous-plane averaging for the SGS enthalpy flux (respectively for the SMD or GRD model) and 

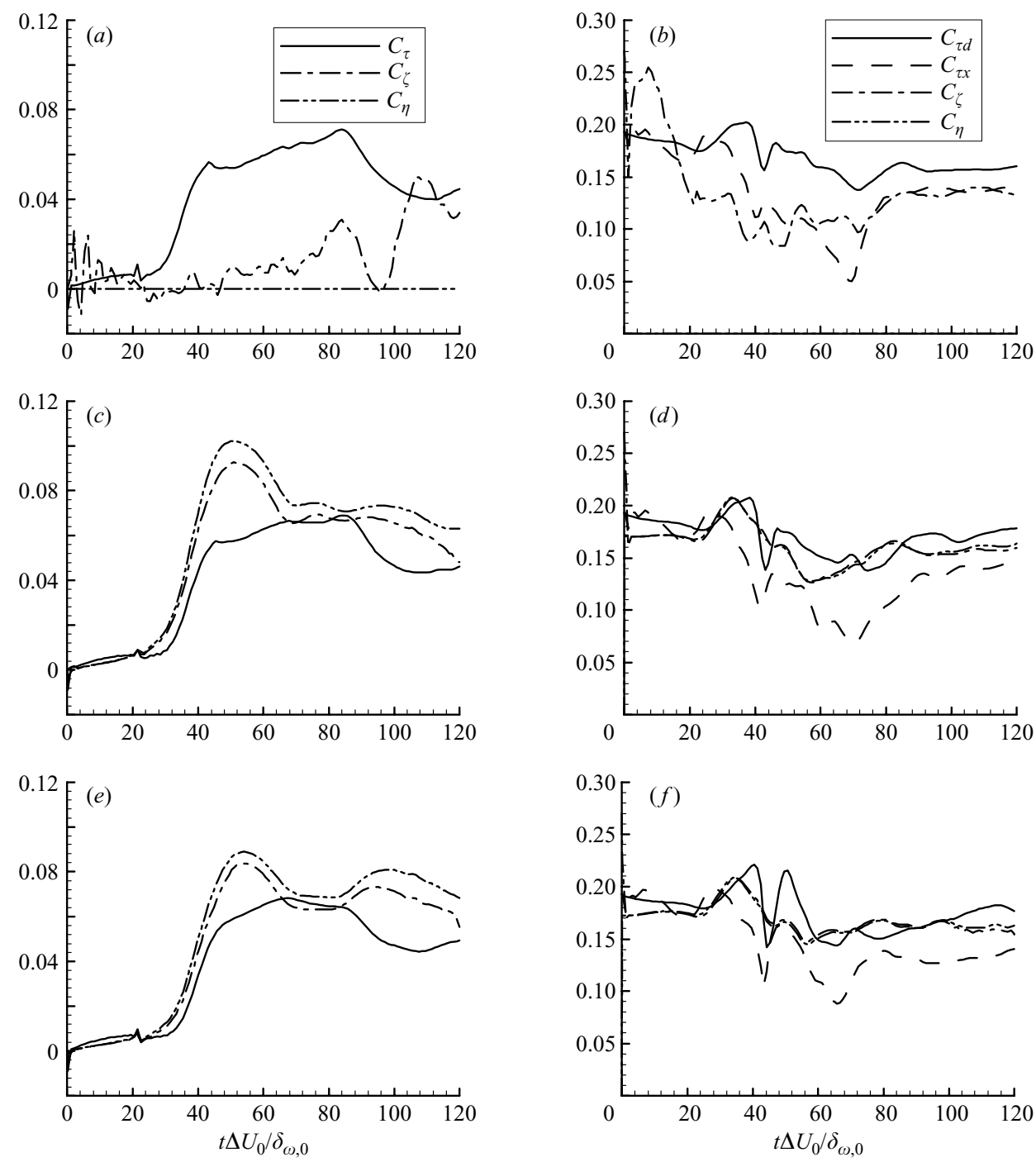

Figure 2. Evolution of the dynamic coefficients in SMD LES (left) and GRD LES (right) for $(a, b)$ SP600 $\left(C_{\eta}=0\right),(c, d)$ TP600a2 with $N_{R}=8$ and $(e, f)$ TP600a5 with $N_{R}=8$.

homogeneous-plane averaging for the SGS species flux (2.48), as these averagings were consistently stable for SP and TP flows. All the dynamic models use $\hat{\Delta}=2 \bar{\Delta}$. Figure 2 presents the domain-average of the SMD and GRD model coefficients for SP600 LES, and for TP600a2 and TP600a5 LES with $N_{R}=8$. For SP600, inherently $C_{\eta}=0$ ( $Y_{V}$ is null). For the SMD model, at the DNS transition times $\left(t^{*}=100\right.$ for SP, $t^{*}=105$ for TP; see table 1 ), the average value of the (non-null) coefficients is 0.052 , 0.059 and 0.064 for SP600, TP600a2 and TP600a5 respectively, slightly lower than the single-coefficient value of 0.072 , which was calibrated at the DNS transition times. As on the DNS database at the DNS transition times, the $C_{\tau}$ value is lower than the average, while the other two coefficient values are above the average (see table 2). For the GRD model, the dynamic coefficients vary dramatically between the first and the second pairing times (for example, in figure $2 d, C_{\tau x}$ decreases from 0.2 to 0.07 between 
$t^{*}=25$ and $t^{*}=70$ ), but have less variation after the second pairing. For SP600, the average value at $t^{*}=100$ of the (non-null) coefficients is 0.1441 . Furthermore, for both TP600a2 and TP600a5, even though at $t^{*}=105$ the four coefficients range in value from 0.1316 to 0.1748 , their average value $(0.1559$ for TP600a 2 and 0.1558 for TP600a5) is close to the calibrated value of 0.1520 , which was obtained on the DNS database at the transitional time. The indications are that one limitation of the SMC and GRC constant coefficients is that they were calibrated at the transition time, whereas they change during the layer evolution. Hence dynamic modelling is necessary to capture the temporal variation of the model coefficients.

Figures $2(d)$ and $2(f)$ suggest that a three-coefficient dynamic gradient model (2.44) for TP flows may be viable, since $\left\langle\left\langle C_{\eta}\right\rangle\right\rangle \simeq\left\langle\left\langle C_{\zeta}\right\rangle\right\rangle$. We devised such a model, denoted GRD3, where $C_{\eta}$ was set equal to the dynamically computed $C_{\zeta}$. The GRD3 model was found to yield results very close to those of the GRD model, therefore only the GRD model results will be shown. The good agreement between the GRD and GRD3 results is most likely a consequence of the correlation between enthalpy and mass fraction occurring in the present simulations and might not have general validity. The $h$-to- $Y_{V}$ correlation is due mainly to two factors: first, (2.18) relates the enthalpy to the mass fractions and (2.21) relates the heat flux to the species mass flux; second, in the present simulations, in (2.21) the transport of energy by the species mass fluxes dominates the heat flux contribution due to the small temperature gradient (see Part 1). With regard to computational performance, the CPU time of the GRD and GRD3 is similar.

The implementation of the SGS-flux models described above considered only the numerical stability of the LES; the accuracy of LES results as compared to DNS is addressed in $\S 5$ and $\S 6$.

\subsection{Comparison to DNS results}

The LES described herein are compared in the following to the DNS results described in detail in Part 1; the DNS cases being considered here are listed in table 1. It should be noted that, although we are primarily interested in the transitional state, by comparing the LES and DNS flow fields at the same physical time, we are also comparing the evolution of the layer by requiring that the layers should evolve to approximately the same transitional state in approximately the same time. We are thereby requiring both temporal equivalence (i.e. the LES transitions at the same time as the DNS) and spatial equivalence (i.e. the LES transitional state be comparable to the DNS state). This is a more rigorous requirement than in spatial flow configurations starting from a turbulent initial condition in LES, where only spatial statistical equivalence is desired.

Comparisons between the filtered DNS database and the LES a posteriori results are performed by calculating the slope $b$ and the correlation factor $\mathscr{R}$ using a least-squares fit of $\mathscr{Y}=b \mathscr{X}$,

$$
b=\frac{\langle\langle\mathscr{X} \mathscr{Y}\rangle\rangle}{\langle\langle\mathscr{X} \mathscr{X}\rangle\rangle}, \quad \mathscr{R}(\mathscr{X}, \mathscr{Y})=\frac{\langle\langle\mathscr{X} \mathscr{Y}\rangle\rangle-\langle\langle\mathscr{X}\rangle\rangle\langle\langle\mathscr{Y}\rangle\rangle}{\sqrt{\left\langle\left\langle\mathscr{X}^{2}\right\rangle\right\rangle-\langle\langle\mathscr{X}\rangle\rangle^{2}} \sqrt{\left\langle\left\langle\mathscr{Y}^{2}\right\rangle\right\rangle-\langle\langle\mathscr{Y}\rangle\rangle^{2}}},
$$

with $\mathscr{Y}$ being the FC-DNS quantity and $\mathscr{X}$ being the LES quantity, defined at the same grid points. For convenience, the tilde and overbar indicating filtered quantities are hereafter omitted. For gas-phase quantities, DNS refers to unfiltered variables whereas FC-DNS and LES refer to filtered variables. 


\begin{tabular}{|c|c|c|c|c|c|c|c|}
\hline \multicolumn{4}{|c|}{ Slopes } & \multicolumn{4}{|c|}{ Correlations } \\
\hline & $\psi_{f}$ & $\begin{array}{c}\tilde{\psi}_{f} \\
(\bar{\Delta}=4 \Delta x)\end{array}$ & $\begin{array}{c}\tilde{\psi}_{f} \\
(\bar{\Delta}=8 \Delta x)\end{array}$ & & $\psi_{f}$ & $\begin{array}{c}\tilde{\psi}_{f} \\
(\bar{\Delta}=4 \Delta x)\end{array}$ & $\begin{array}{c}\tilde{\psi}_{f} \\
(\bar{\Delta}=8 \Delta x)\end{array}$ \\
\hline$S_{I, d}$ & 0.88 & 0.92 & 0.96 & $S_{I, d}$ & 0.96 & 0.98 & 0.99 \\
\hline$S_{I I, 1, d}$ & 0.57 & 0.71 & 0.84 & $S_{I I, 1, d}$ & 0.76 & 0.88 & 0.95 \\
\hline$S_{I I, 2, d}$ & 0.52 & 0.70 & 0.87 & $S_{I I, 2, d}$ & 0.67 & 0.83 & 0.93 \\
\hline$S_{I I, 3, d}$ & 0.39 & 0.54 & 0.73 & $S_{I I, 3, d}$ & 0.59 & 0.75 & 0.86 \\
\hline$S_{I I I, d}$ & 0.83 & 0.87 & 0.90 & $S_{I I I, d}$ & 0.93 & 0.95 & 0.96 \\
\hline
\end{tabular}

\section{A priori analysis of interpolation error in the filtered source terms}

In the a priori analysis of Part 1, all modelling was performed on the DNS (fine) grid. The flow field is calculated only at the grid points, and must be interpolated to the drop locations in order to provide the far-field conditions $\psi_{f}$ needed to compute the drop source terms $\left(S_{d}\right)$ through (2.27). Foreseeing computations on the LES grid, Part 1 included an assessment of the error in computing $S_{d}$ when the unfiltered variables $(\psi)$ are replaced by the filtered variables $(\tilde{\psi})$, and of the resulting errors in the FSTs $(\bar{S})$ which are calculated from $S_{d}$ through (2.31). Given that the LES grid is coarser than the DNS grid, it is appropriate to inquire what further effect the grid coarsening will have on $S_{d}$ and on $\bar{S}$. To this end, we here calculate $S_{d}$ and $\bar{S}$ using either $\psi_{f}$ or $\tilde{\psi}_{f}$ interpolated on the coarser LES grid, denoted $\psi_{f, \text { LES }}$ or $\tilde{\psi}_{f, \text { LES }}$. The calculation with $\psi_{f, \text { LES }}$ contains only interpolation errors, while that with $\tilde{\psi}_{f, \text { LES }}$ additionally contains errors in the drop far field (lack of SGSs). Therefore, to isolate interpolation errors, the $\tilde{\psi}_{f, \text { LES }}$ calculation is compared to DNS-grid calculations with $\tilde{\psi}_{f, \mathrm{DNS}}$; that is, $\tilde{\psi}$ is first obtained on the DNS grid, and then $\tilde{\psi}_{f, \mathrm{LES}}$ and $\tilde{\psi}_{f, \mathrm{DNS}}$ are obtained from interpolation on the LES and DNS grids, respectively. In table 3 is presented the comparison of DNS-grid and LES-grid $S_{d}$ using least-squares-fit slopes and correlations ((3.3) applied at drop locations). All the slopes are less than unity, meaning that $S_{d}$ is over-predicted. As observed in Part 1, $S_{I I, 3, d}$ is the worst predicted, due to $u_{3}$ having the smallest magnitude of the three velocity components. The interpolation error (deviation of slope and correlation from unity) is seen to be largest for $S_{d}$ based on the unfiltered variables and smallest for $S_{d}$ based on filtered variables at the larger filter width. The explanation of the error decrease as the filter width increases is that filtering smooths the flow, thereby reducing the gradients and hence the interpolation errors. The error in $S_{I I, 3, d}$ of about $27 \%$ at $\bar{\Delta}=8 \Delta x_{\mathrm{DNS}}$, which is the filter width to be used for the LES in the present study, is somewhat lower than the error in modelling $\psi_{f}$ by $\tilde{\psi}_{f}$ (see Part 1).

Combining the interpolation and computational-drop-modelling error, in figure 3 we compare $\bar{S}\left(\tilde{\psi}_{f}, N_{R}\right)$ for various values of $N_{R}$ on the LES grid to $N_{R}=1$ on the DNS grid; $\bar{S}$ on the DNS grid uses the physical drops whereas $\bar{S}$ on the LES grid uses computational drops. Similar to $S_{d}, \bar{S}$ is over-predicted, the prediction of $\bar{S}$ is better at the larger filter width and $\bar{S}_{I I, 3}$ is the worst predicted component. $\bar{S}$ shows a clear degradation of accuracy with increasing $N_{R}$, although the degradation is not 

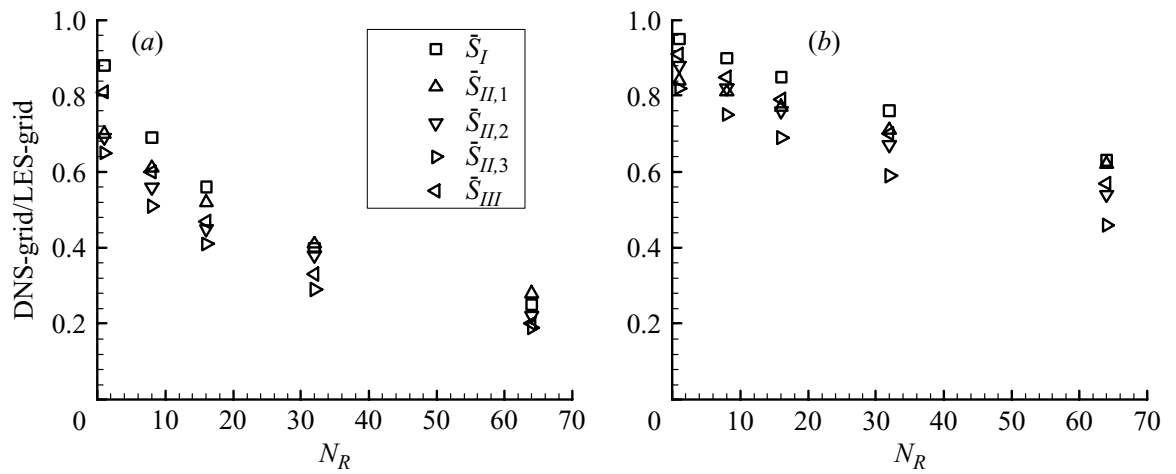

FiguRE 3. A priori analysis of interpolation error in $\bar{S}$ : TP600a2 at $t^{*}=105$. Slopes (DNS-grid/ LES-grid) from least-squares fits at LES grid points. DNS-grid (LES-grid) $\bar{S}$ uses $\tilde{\psi}_{f}$ obtained by interpolating $\tilde{\psi}$ on DNS (LES) grid and $N_{R}=1\left(N_{R} \geqslant 1\right) ; \tilde{\psi}$ results from filtering $\psi$ on DNS grid using filter width $\bar{\Delta}$. (a) $\bar{\Delta}=4 \Delta x_{\mathrm{DNS}}$ and $(b) \bar{\Delta}=8 \Delta x_{\mathrm{DNS}}$.

\section{SGS model}

SMC Smagorinsky, constant coefficient

SMD Smagorinsky, dynamic coefficient $(\hat{\Delta}=2 \bar{\Delta})$

GRC Gradient, constant coefficient

GRD Gradient, dynamic coefficient $(\hat{\Delta}=2 \bar{\Delta})$

SSC Scale-similarity, constant coefficient $(\hat{\Delta}=\bar{\Delta})$

$\begin{array}{lll}\delta_{m} / \delta_{\omega, 0} & \delta_{m} / \delta_{\omega, 0} \\ \text { at } t_{\text {trans }}^{*} & t_{\text {peak }}^{*} & \text { at } t_{\text {peak }}^{*}\end{array}$

2.480

2.390

2.486

2.146

2.279

95

2.489

$90 \quad 2.498$

$95 \quad 2.543$

$90 \quad 2.298$

$95 \quad 2.323$
CPU-hours 3.0

5.9

TABLE 4. LES runs corresponding to DNS case SP600. For all cases, grid is $72 \times 80 \times 44$; see table 1 for other initial conditions. DNS transition time $t_{\text {trans }}^{*}=100$. Coefficient values for SGS models: $C_{\mathrm{SM}}=0.072, C_{\mathrm{YO}}=0.314(\mathrm{SMC}) ; C_{\mathrm{GR}}=0.15(\mathrm{GRC}) ; C_{\mathrm{SS}}=1.996$ (SSC). CPU-hours: aggregate over parallel processors on SGI Origin 2000.

as severe at the larger filter width. This behaviour was also observed in Part 1, in the a priori FST modelling on the DNS grid, and was there attributed to the complex interaction, as the filter width is increased, between the reduction in the accuracy with which the unfiltered flow field is represented and the increase in accuracy due to the larger number of drops contained in the filtering volumes. It is not possible to quantify a priori how the decrease in accuracy with increasing $N_{R}$ will affect the LES; such an evaluation will be made in $\S 6$.

\section{Single-phase-flow LES results}

The usefulness of the single-phase case is to develop candidate SGS-flux models for TP flows, since, within statistical significance, these models were found to perform similarly in a priori studies on the SP600 and TP600a2 DNS databases. Compared to TP LES, SP LES has reduced computational costs and does not require FST modelling. SP LES were here performed with the constant-coefficient and dynamic SGS-flux models listed in table 4, which also lists the CPU time. Compared to the DNS (table 1), the LES takes a factor of 179 to 351 less time. This reduction is less than the factor of 512 that would result from the grid coarsening (and associated 

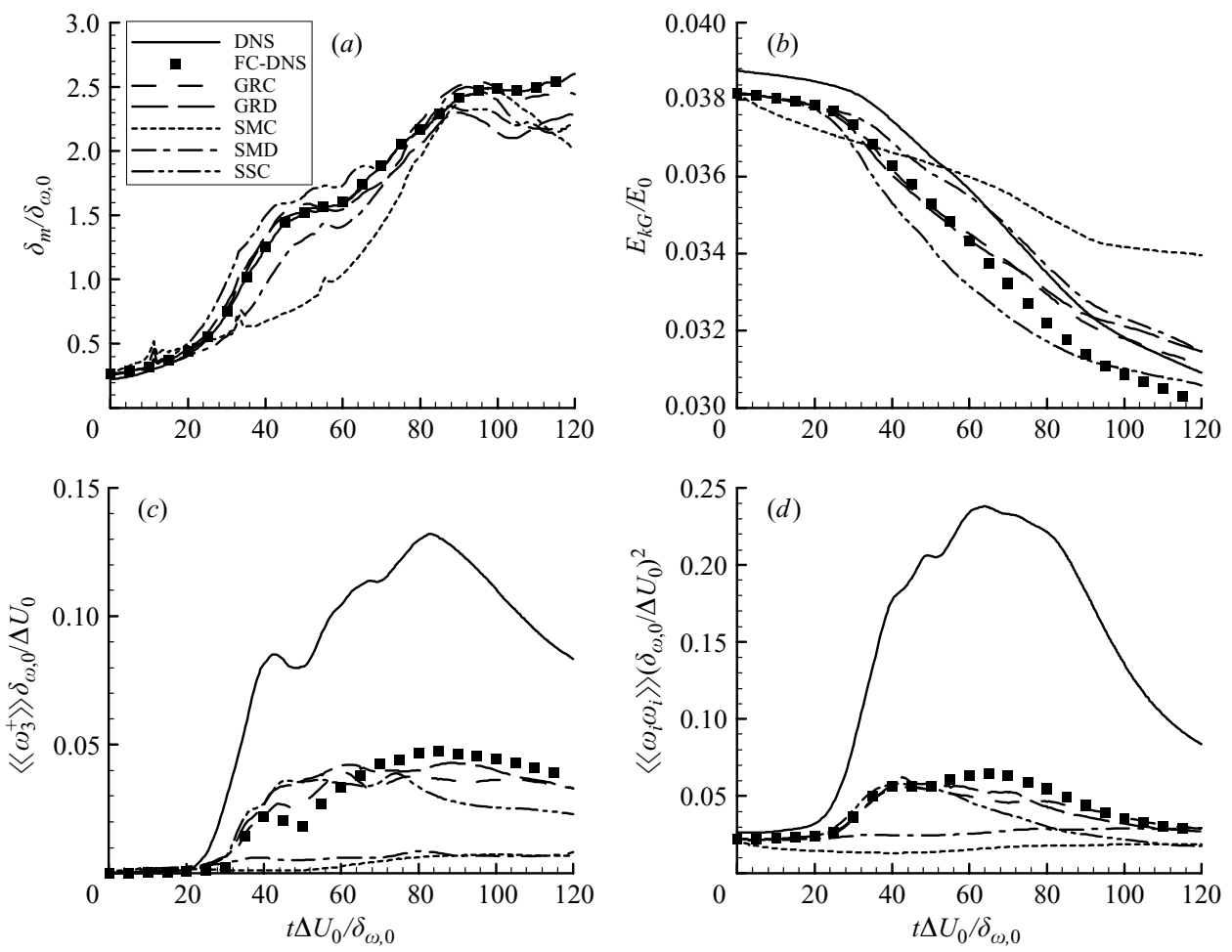

FIGURE 4. Time evolution of non-dimensional global quantities for SP600: $(a)$ momentum thickness, $(b)$ resolved kinetic energy, $(c)$ average positive spanwise vorticity and $(d)$ average enstrophy. DNS; FC-DNS; LES listed in table 4. $E_{0}=1392.2 \mathrm{~J}$ is the initial total energy in the domain.

increase in time step) alone, the larger CPU time being due to the overhead of computing the SGS-flux models. The SMC and GRC models, which use already available gradients, are the least expensive, while the SSC model, which involves refiltering, is the most expensive. The GRD and SMD models also require re-filtering, but are faster than the SSC model, despite utilizing a larger test-filter width, since they only require refiltering once per time step (in computing the coefficients at the beginning of each time step), whereas the SSC model requires refiltering four times per time step (at each stage of the time-integration). The performance of the different models as it pertains to the quality of the LES is discussed below.

\subsection{Evolution of the global quantities}

Since the goal of the present LES is to replicate the DNS results, plotted in figure 4 is the temporal evolution of various global quantities from LES using the five different SGS-flux models listed in table 4; these results are to be compared to the DNS and FC-DNS results. In considering the momentum thickness $\delta_{m}(3.1)$ in figure $4(a)$, we observe that all layers show sustained growth until approximately the time of the second pairing, after which $\delta_{m}$ diminishes. (The DNS and FC-DNS curves overlap.) However, the SMC model has an initially reduced rate of growth compared to all the other models, which show good agreement with the FC-DNS results. When the entire evolution is considered, the best agreement comes from the GRC model; the 
GRD model agrees with the GRC model in the early stages of the layer evolution. The values of $\delta_{m}$ at the DNS transition time $t_{\text {trans }}^{*}=100$ are listed in table 4 , which shows the GRC and SMC model $\delta_{m}$ predictions to be within $0.2 \%$ of the DNS value of 2.485 . The other models are not as accurate in predicting $\delta_{m}$, being $4 \%$ (SMD) to $14 \%$ (GRD) too low. For all SGS-flux models, the time of the peak in $\delta_{m}$ after the second pairing, $t_{\text {peak }}^{*}$, also listed in table 4 , occurs sooner for LES than for DNS and has a smaller $\delta_{m}$. The best peak $\delta_{m}$ prediction comes from the SMC model, while the worst is that of the GRD model which has an error of $7 \%$ in $\delta_{m}$. For the DNS, consideration of an ensemble of criteria is required to determine whether transition has been attained at any particular time (see $\S 3.1$ ), whereas no such criteria exist for LES. In this study, comparisons of the LES to the FC-DNS will be made at the DNS transition time, $t_{\text {trans }}^{*}$ in table 1 , and, based on the flow characteristics, an assessment will be made of whether the LES has achieved transition at that time.

Depicted in figure $4(b)$ is the resolved kinetic energy $E_{k G}=\int_{\text {domain }} \rho\left(u_{i} u_{i} / 2\right) \mathrm{d} V$. Notably, the kinetic energy represents a small fraction (between $3 \%$ and $4 \%$ ) of the total energy in the system $E_{0}$. Here, the difference between the DNS and FC-DNS represents the SGS kinetic energy $\tau_{i i} / 2$, and it is seen that, even at the transition time, the SGS kinetic energy is a small fraction of the resolved kinetic energy (although a larger portion, about $14 \%$, of the turbulent kinetic energy resides in the SGS). Nevertheless, SGS-flux models were found to be necessary in order to compute flows on the LES grids (the code with no SGS model crashed), this being evidence that the high accuracy of the numerical method does not allow under-resolved computations. The $E_{k G}$ plot shows that the initial rate of decay for the SMC model is greater than that for the other models, indicating that the SMC model is here the most dissipative. However, whereas the other models begin at rollup $\left(t^{*} \sim 20\right)$ a period of increased decay rate, as does the FC-DNS, this period is delayed for the SMC model until $t^{*} \sim 60$, and this model retains the largest $E_{k G}$, indicating that it lacks the small resolved scales (SRSs) that would be responsible for turbulent dissipation. The SSC model shows the largest overall kinetic energy loss. We note that although all SGS models show global energy dissipation, the Smagorinsky model by construction guarantees positive local dissipation $\left(\tau_{i j} \partial \tilde{u}_{i} / \partial x_{j}>0\right.$ for $\chi>0$, see figure 2$)$; the gradient and scale-similarity models inherently allow for local backscatter.

Depicted in figures $4(c)$ and $4(d)$ are two vorticity measures for the resolved fields: the positive spanwise vorticity $\left\langle\left\langle\omega_{3}^{+}\right\rangle\right\rangle$, which measures the SRS activity since the initial $\omega_{3}$ is negative; and the enstrophy $\left\langle\left\langle\omega_{i} \omega_{i}\right\rangle\right\rangle$, which measures stretching and tilting, an important mechanism for turbulence production. For both measures, the FC-DNS value is as small as one-third to one-fourth of the DNS value, due to the removal of SGSs by the filtering operation. The FC-DNS curves qualitatively mimic the DNS ones, in growing after rollup $\left(t^{*} \sim 20\right)$, reaching a peak at approximately the time of the second pairing $\left(t^{*} \sim 80\right)$ and then decaying. In examining the LES results, we note that for both SMC and SMD models, there is little activity, in agreement with the initially overly dissipative aspect seen in figure $4(b)$, whereas the other models qualitatively and sometimes quantitatively match the FC-DNS results. The best agreement comes from the GRD model, with the GRC model also performing well. The SSC model performs comparably to the GRC and GRD models in the early part of the layer evolution, but has earlier and greater vorticity decay, in agreement with the results of figure $4(b)$.

The performance of the SGS-flux models is further assessed at the DNS transitional time by examining contour plots, energy spectra, homogeneous-plane statistics and global flowfield correlations. 

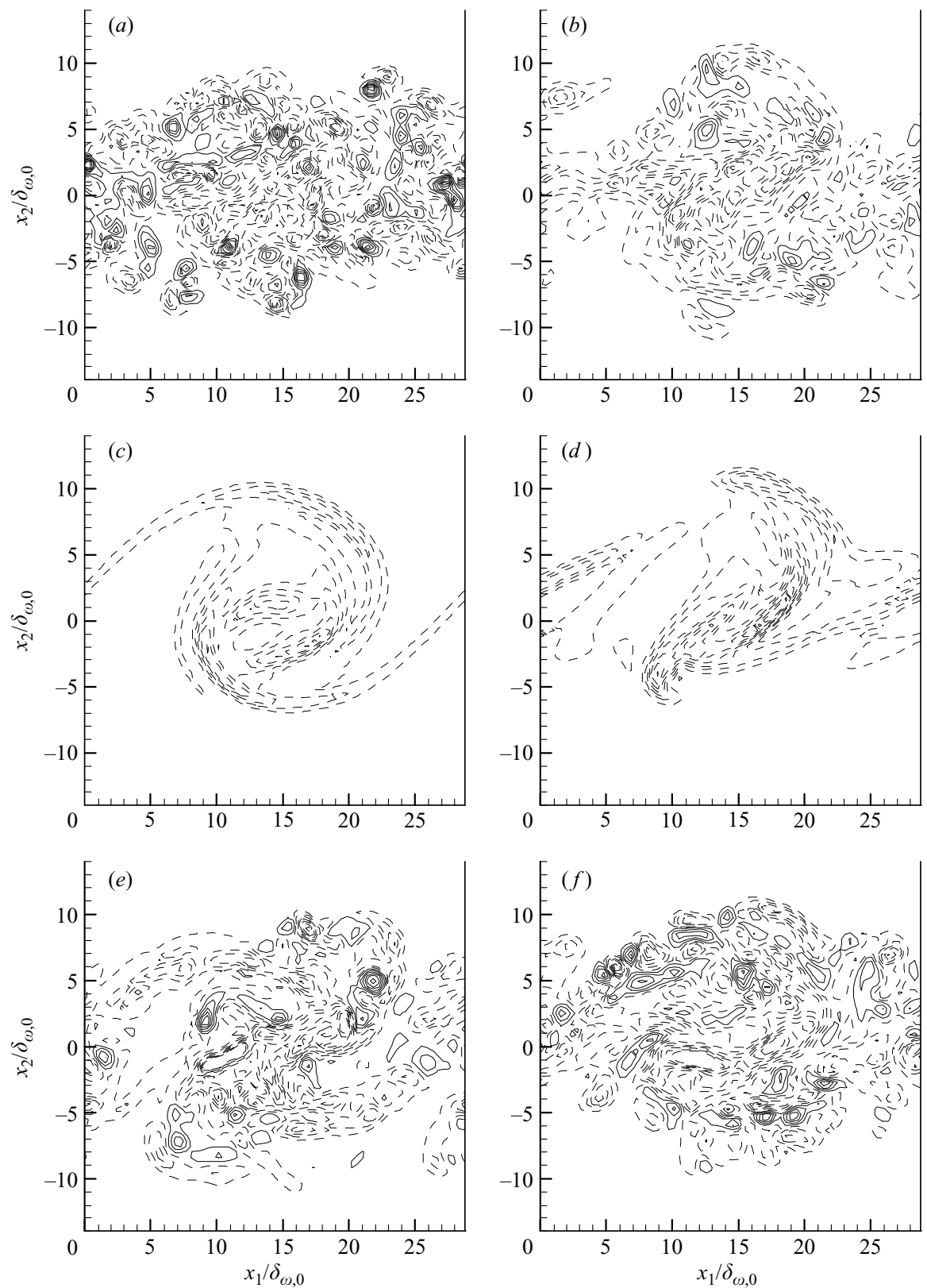

FIGURE 5. Spanwise vorticity (non-dimensional) for SP600 at $t^{*}=100$, in the between-the-braid plane $\left(x_{3} / L_{3}=0.5\right)$ : $(a)$ FC-DNS and $(b-f)$ LES using $(b)$ SSC, $(c)$ SMC, $(d)$ SMD, $(e)$ GRC and $(f)$ GRD SGS-flux model. Dashed lines are used for negative values. Contour levels range from -0.579 to 0.524 in increments of 0.0788 (15 levels).

\subsection{Flow visualizations}

To complement the global vorticity measures plotted in figure 4, in figures 5 and 6 are contour plots of the spanwise and streamwise vorticity, $\omega_{3}$ and $\omega_{1}$, respectively, at $t^{*}=100$. In comparing the FC-DNS and LES results, clearly the SMC and SMD LES are almost entirely devoid of SRSs, although both display the largest scale ultimate spanwise vortex in figure 5. This explains figure 4 which showed the good growth 

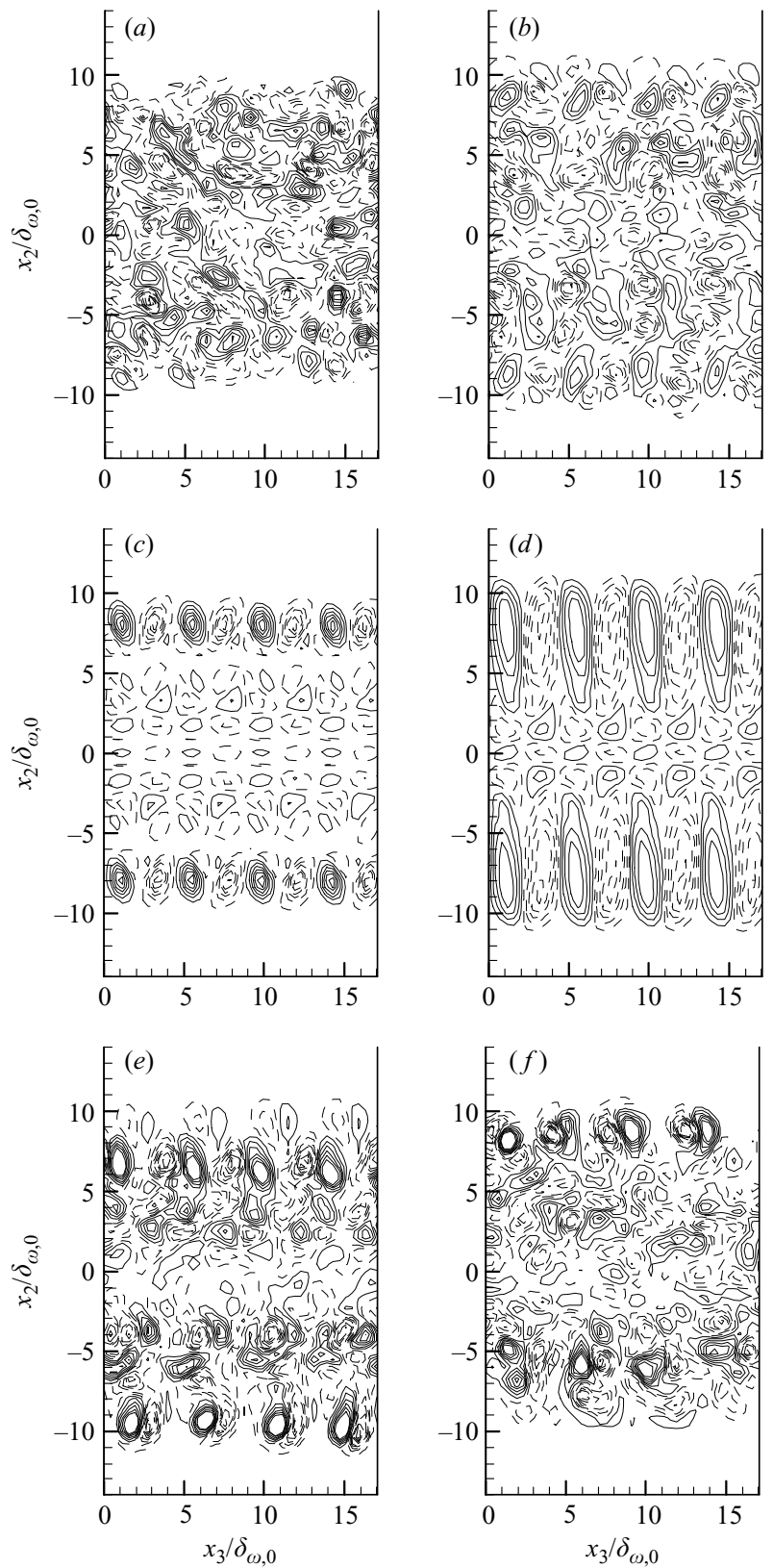

FIGURE 6. Streamwise vorticity (non-dimensional) for SP600 at $t^{*}=100$, in the mid-plane $\left(x_{1} / L_{1}=0.5\right)$ : $(a)$ FC-DNS and $(b-f)$ LES using $(b)$ SSC, $(c)$ SMC, $(d)$ SMD, $(e)$ GRC and $(f)$ GRD SGS-flux model. Contour levels range from -0.631 to 0.436 in increments of 0.076 (15 levels).

of these layers (due to entrainment and manifested by the largest scale vortices), but minimal positive spanwise vorticity and enstrophy generation. In considering $\omega_{1}$ in figure 6, both the SMC and SMD models show only spanwise structures with a wavelength that is one-fourth of the domain length. These structures are in fact those initially imposed, and no SRS structures have emerged. On the other hand, the SSC, 

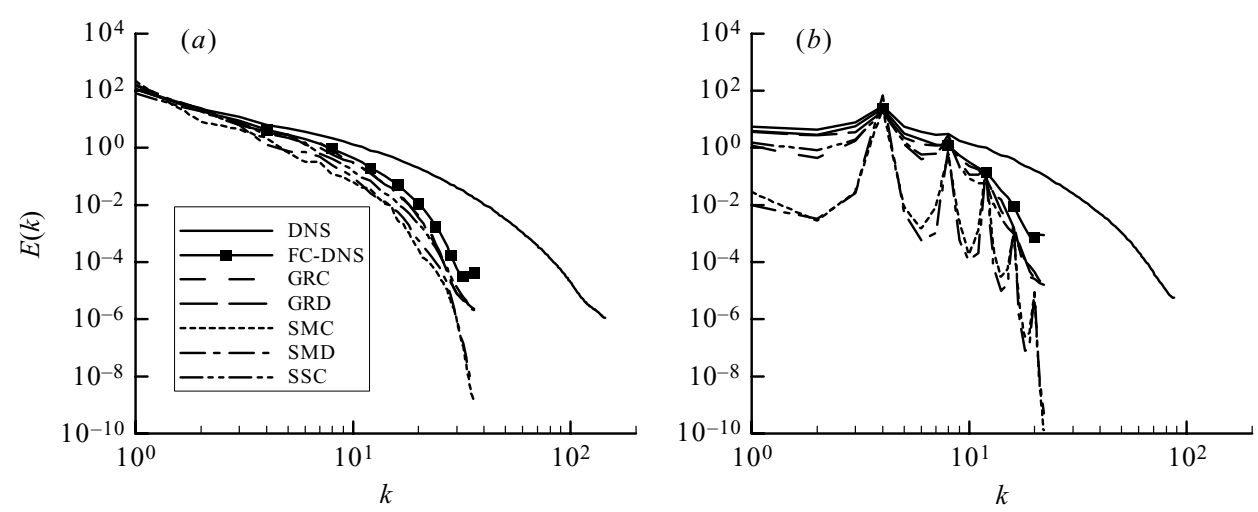

FIGURE 7. Energy spectra of $u_{1}$ for SP600 at $t^{*}=100:(a)$ streamwise and $(b)$ spanwise. Energy in $\mathrm{m}^{2} \mathrm{~s}^{-2}$.

GRC and GRD models lead to $\omega_{3}$ and $\omega_{1}$ flow fields that look quite similar to the FC-DNS. In contrast to the SMC and SMD LES, the other models show little or no memory of the initial conditions in $\omega_{1}$, and exhibit a variety of structure sizes. The lack of SRSs for the SMC and SMD LES leads to questioning whether a transitional state was truly achieved at the DNS transitional time. This issue can be clarified by examining the energy spectra.

\subsection{One-dimensional spectra of velocity components}

Figure 7 displays one-dimensional streamwise and spanwise energy spectra, which show the energy content of each wavenumber $(k)$ in the flow; the $u_{1}$ spectra depicted in figure 7 are similar to those for $u_{2}$ and $u_{3}$. With respect to the DNS, the FC-DNS is seen to be an excellent approximation at the largest scales, but at smaller scales it increasingly departs from the DNS and exhibits an accumulation of energy at the SRS, these aspects being a manifestation of the filtering. Comparing the LES spectra to those of the FC-DNS, the gradient-model spectra are closest, followed by the SSC-model spectra. For the streamwise spectra, the SSC, GRC and GRD models exhibit a smooth aspect and closely follow the FC-DNS; they display smaller amounts of energy than the FC-DNS at the largest wavenumber due to the effect of the SGS models. The SMC and SMD streamwise spectra exhibit small peaks and display smaller amounts of energy than the FC-DNS with increasing $k$, while around and past the filter cut-off, the amount of energy is orders of magnitude smaller than for the FC-DNS. For the spanwise spectra, the SMC and SMD models show sharp peaks, corresponding to structures in the spanwise direction (see figure 6) which persist past the initial perturbation. These peaks are not observed for the DNS, the FC-DNS and the GRD models; for the GRC and SSC models, these peaks are much smaller and only occur at $k=8$. (All spanwise spectra, including the DNS, have a peak at $k=4$ corresponding to the spanwise forcing.) Additionally, it is apparent that for the SMC and SMD models even the energy at the largest scale does not duplicate that of the FC-DNS, being smaller by more than an order of magnitude. Therefore, the Smagorinsky model, while permitting pairing of the initial spanwise vortices and growth of the layer, has actually not reached transition to turbulence. Furthermore, dynamic modelling does not lead to noticeable improvement in the spectra yielded by the Smagorinsky model. The information in figures 4-7 is entirely consistent in 
that it is evident that the Smagorinsky model is incapable of reproducing transition, in contrast to the attainment of transition by the gradient and SSC models.

It should be noted that the present test of the Smagorinsky model is stringent but appropriate in that it involves only considerations of the flow in the absence of solidwall boundary conditions and initially prescribed turbulence. In most engineering applications where the Smagorinsky (or the similar Prandtl mixing length) model is used and a flow with seemingly turbulent features is created, the simulations are performed in configurations where solid walls are present or turbulent initial conditions are prescribed. Boundary conditions at solid walls will create vorticity which, when transported to the core of the flow, will act as a surrogate turbulence. However, the present evaluations of the Smagorinsky model show that, in the absence of walls, such surrogate turbulence features are not produced. Therefore, results from existing engineering simulations may be seriously misleading regarding the LES potential of the Smagorinsky model.

\subsection{Homogeneous-plane gas-phase statistics}

Next, the LES are compared from the standpoint of statistics computed on homogeneous $\left(x_{1}, x_{3}\right)$-planes. Figure 8 shows the planar averages and the fluctuations relative to the planar averages, for the velocity components and for $T$; similar results were calculated for $\rho$ and $p$ but are not shown. The DNS and FC-DNS results overlap for the velocity averages and the $T$ fluctuations, and are close for the $u_{1}$ and $u_{2}$ fluctuations, indicating that planar quantities are generally insensitive to the existence of SRS structures. This indication is further borne out by comparison of the LES velocity planar quantities, which do not reveal the qualitative differences among the models that were observed in the contour plots in figure 5. In fact, on a planar basis, the SMD and SMC models perform as well as or better than the other models in predicting the velocity components, despite their clear deficiencies in predicting global rotational aspects of the flow (see figures $4 c$ and $4 d$ ). All models give comparably good predictions for the $u_{1}$ averages. Differences among the models are more apparent in the averages of $u_{2}$ and $u_{3}$. For the $u_{2}$ average, the SMC model is the best, although exhibiting some oscillations, while the SSC model is qualitatively incorrect; for the $u_{3}$ average, the GRC model is obviously the worst, but the other models perform similarly to one another. All models under-predict the fluctuations of $u_{3}$, which, since it has the smallest magnitude of the three velocity components, is the most difficult to predict; here the GRD model performs best, followed by the GRC and SSC models, although none of the models captures the location of both peaks on the two sides of the centreline. For the fluctuations of $u_{1}$ and $u_{2}$, the GRC and GRD models are similar, and generally outperform the SMD model, which in turn is better than the SMC model; the SSC model result lies between the GRD and SMD values.

Considering the temperature in figure 8 , the models predict a small range of variation in the average (less than 2\%); however, except for the SMD model, which has minimal variation across the layer, each range is larger than that of the DNS and the FC-DNS. The GRD has the best $T$ average, while the SMD has the best fluctuations; the SMC is the worst in both respects. The constant-coefficient versions, SMC and GRC, give qualitatively incorrect results for the $T$ average; the GRC model, while an improvement over the SMC model, still has a dip in the temperature in the middle of the domain which is not present in the DNS or FC-DNS. The SSC model tends to over-predict the centreline average of $T$, but does quite well in predicting the fluctuations. Both the GRC and GRD models over-predict the fluctuations in $T$, although not as severely as does the SMC model. The models perform similarly in 

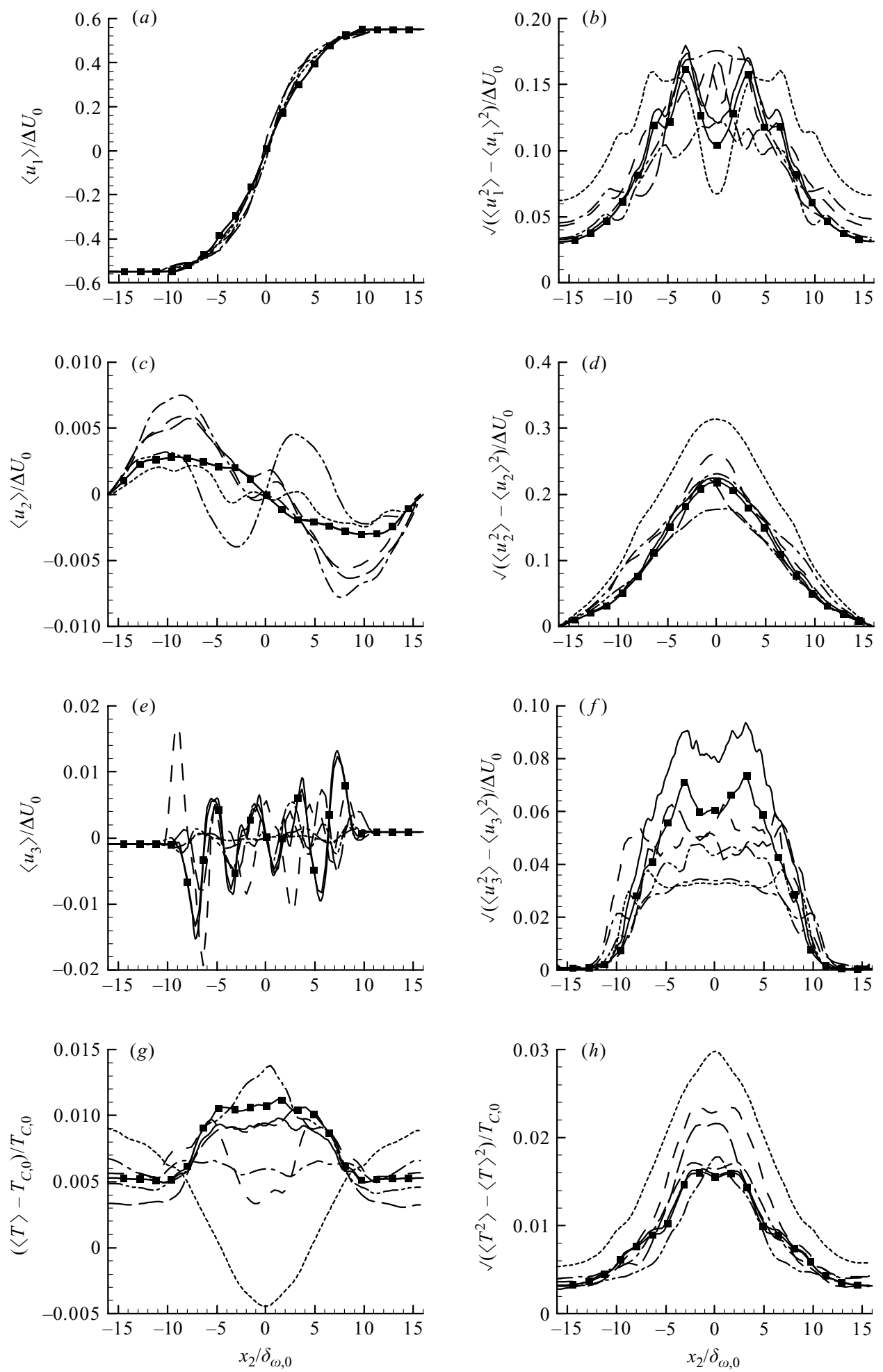

FIGURE 8. Homogeneous-plane statistics of gas-phase quantities for SP600 at $t^{*}=100:(a, b) u_{1}$, $(c, d) u_{2},(e, f) u_{3}$ and $(g, h) T$. See figure 7 for legend. Averages (left) and fluctuations (right). 


\begin{tabular}{|c|c|c|c|c|c|c|c|c|c|c|c|}
\hline \multicolumn{6}{|c|}{ Slopes } & \multicolumn{6}{|c|}{ Correlations } \\
\hline & SMC & SMD & GRC & GRD & $\mathrm{SSC}$ & & SMC & SMD & GRC & GRD & $\mathrm{SSC}$ \\
\hline$u_{1}$ & 0.97 & 0.96 & 0.98 & 0.96 & 0.97 & $u_{1}$ & 0.99 & 0.98 & 0.99 & 0.99 & 0.98 \\
\hline$u_{2}$ & 0.58 & 0.69 & 0.74 & 0.89 & 0.94 & $u_{2}$ & 0.89 & 0.78 & 0.85 & 0.84 & 0.82 \\
\hline$u_{3}$ & 0.11 & 0.14 & 0.03 & 0.07 & -0.02 & $u_{3}$ & 0.07 & 0.08 & 0.03 & 0.06 & -0.01 \\
\hline$\rho$ & 1.00 & 1.00 & 1.00 & 1.00 & 1.00 & $\rho$ & 0.98 & 0.97 & 0.97 & 0.96 & 0.98 \\
\hline$T$ & 1.00 & 1.00 & 1.00 & 1.00 & 1.00 & $T$ & 0.82 & 0.86 & 0.88 & 0.91 & 0.88 \\
\hline$p$ & 1.00 & 1.00 & 1.00 & 1.00 & 1.00 & $p$ & 0.99 & 0.98 & 0.98 & 0.97 & 0.98 \\
\hline$\omega_{1}$ & 0.15 & 0.16 & 0.03 & 0.09 & -0.06 & $\omega_{1}$ & 0.12 & 0.16 & 0.04 & 0.09 & -0.04 \\
\hline$\omega_{2}$ & 0.27 & 0.21 & 0.07 & 0.15 & -0.07 & $\omega_{2}$ & 0.18 & 0.19 & 0.06 & 0.12 & -0.05 \\
\hline$\omega_{3}$ & 0.64 & 0.59 & 0.42 & 0.38 & 0.49 & $\omega_{3}$ & 0.42 & 0.40 & 0.35 & 0.32 & 0.34 \\
\hline
\end{tabular}

TABLE 5. Slopes (exact/model) and correlations from least-squares fit of FC-DNS to LES flow-field quantities for LES runs listed in table 4: SP600 at $t^{*}=100$.

predicting the $\rho$ and $p$ fluctuations as they do in predicting the $T$ fluctuations, but predict the $\rho$ and $p$ averages better than they predict the $T$ averages. The SMD gives the best predictions for both the averages and fluctuations of $\rho$ and of $p$. All of the constant-coefficient models (SMC, GRC, SSC) show some deficiency in calculating at least one plane-average quantity, indicating that dynamic models may ultimately be necessary.

\subsection{Flow-field correlations}

To perform a more quantitative comparison of the LES results, these were correlated with the FC-DNS using (3.3); the results are summarized in table 5. These correlations are meant to mimic an equivalent comparison of LES with possible experimental data, which is here portrayed by the FC-DNS. Of the velocity components, the streamwise velocity $u_{1}$ has the best slopes and correlations (closest to unity) while the spanwise velocity $u_{3}$ has the worst, consistent with the planar results (figures $8 e$ and $8 f$ ). The $T$ slopes are unity for all models, and the correlations are high, even for the SMC model, despite its poor planar averages (figure $8 g$ ). For all models, the vorticity components are not as well-correlated to the FC-DNS as the velocity components, which is expected since these derivative quantities are more sensitive to errors. The SMC and SMD models appear to give the best slopes and correlations for the vorticity components, in contradiction to the observations in figures 5 and 6 , indicating that correlations may not be a reliable diagnostic of the detailed structure of the flow, which is of ultimate interest if mixing is studied. The larger vorticity correlations for the SMC and SMD models are attributed to the persistence of the initial conditions (see figure 7) that allow a stronger coherent vortex while the SRS formation is much reduced (figure $4 c$ ). Thus, comparisons of experiments and simulations, for the purpose of model validation, must be made from a different viewpoint than that of global correlations.

\subsection{Summary of SP LES results}

The SP600 LES have afforded the opportunity to study the SGS-flux models independently of drop-related effects. Also, they allowed the testing of the DNS-calibrated coefficients obtained in the a priori study of Part 1 . It was found that the a priori study led on one hand to overly optimistic conclusions, in that the calibrated coefficients were not consistently numerically stable (e.g. the SSC model with $\hat{\Delta}=2 \bar{\Delta}$ ). On the other hand, the gradient and scale-similarity models that were found accurate in the 


\begin{tabular}{|c|c|c|c|c|c|c|}
\hline SGS model & $N_{R}$ & $N_{c d, 0}$ & $\begin{array}{l}\delta_{m} / \delta_{\omega, 0} \\
\text { at } t_{\text {trans }}^{*}\end{array}$ & $t_{\text {peak }}^{*}$ & $\begin{array}{l}\delta_{m} / \delta_{\omega, 0} \\
\text { at } t_{\text {peak }}^{*}\end{array}$ & CPU-hours \\
\hline SMD & 1 & 2993630 & 2.227 & 95 & 2.389 & 100 \\
\hline SMD & 8 & 374203 & 2.223 & 95 & 2.387 & 20 \\
\hline SMD & 16 & 187101 & 2.226 & 95 & 2.389 & 12 \\
\hline SMD & 32 & 93550 & 2.224 & 95 & 2.385 & 10 \\
\hline SMD & 64 & 46775 & 2.225 & 95 & 2.385 & 8 \\
\hline GRD & 1 & 2993630 & 2.084 & 100 & 2.168 & 113 \\
\hline GRD & 8 & 374203 & 2.102 & 100 & 2.174 & 20 \\
\hline GRD & 16 & 187101 & 2.080 & 100 & 2.180 & 12 \\
\hline GRD & 32 & 93550 & 2.085 & 95 & 2.173 & 9 \\
\hline GRD & 64 & 46775 & 2.083 & 95 & 2.153 & 8 \\
\hline $\mathrm{SSC}$ & 1 & 2993630 & 2.318 & 100 & 2.334 & 99 \\
\hline $\mathrm{SSC}$ & 8 & 374203 & 2.270 & 100 & 2.291 & 19 \\
\hline $\mathrm{SSC}$ & 16 & 187101 & 2.252 & 100 & 2.274 & 13 \\
\hline $\mathrm{SSC}$ & 32 & 93550 & 2.235 & 100 & 2.252 & 11 \\
\hline $\mathrm{SSC}$ & 64 & 46775 & 2.195 & 100 & 2.230 & 9 \\
\hline
\end{tabular}

TABLE 6. LES runs corresponding to DNS case TP600a2. For all cases, grid is $72 \times 80 \times 44$; see table 1 for other initial conditions. $N_{c d, 0}=N_{d, 0} / N_{R}$ is the initial number of computational drops. DNS transition time $t_{\text {trans }}^{*}=105$. CPU-hours: aggregate over parallel processors on SGI Origin 2000.

a priori study, when numerically stable, did perform much better than the Smagorinsky model, which exhibited an inability to achieve transition. It was shown that the LES data need to be studied from several different viewpoints (such as global measures and correlations, contour plots, spectra and homogeneous-plane statistics), in order to determine whether they replicate the DNS results. In particular, global correlations and homogeneous-plane statistics were insensitive to the SRS activity, and may lead to an erroneous assessment of the accuracy of an LES model. In contrast, globally averaged vorticity measures were much more revealing of SRS structure, consistent with contour plots and velocity spectra. Having evaluated the SGS-flux models in the absence of drops, we now examine their performance in TP LES, where the flow field and drops interact, and thus we additionally investigate the adequacy of the proposed computational-drop models.

\section{Two-phase-flow LES results}

TP LES were performed for cases TP600a2 and TP600a5 listed in table 1 using the same SGS-flux models as for SP600 discussed above, namely the GRC, SSC, GRD and SMD SGS-flux models (described in §2.4); however, the SMC model was not used owing to its poor accuracy for SP600. The SMD model showed little improvement over the SMC model, but is retained here due to its popularity with TP LES researchers, such as listed in the Introduction. The GRC model also was eliminated due to its numerical instability for TP600a5. Therefore, the only remaining SGS-flux models were SSC, SMD and GRD (SSC using $\hat{\Delta}=\bar{\Delta}$, SMD and GRD using $\hat{\Delta}=2 \bar{\Delta}$ ). LES were conducted using these three models and the same FST model (2.32) with $N_{R}$ of $1,8,16,32$ or 64 ; the simulations are listed in tables 6 and 7. The TP LES with any of the SSC, SMD and GRD models use comparable amounts of CPU time, unlike for SP600 (table 4) where the SSC CPU time was noticeably larger. The CPU time declines as the number of computational drops is 


\begin{tabular}{lrrrrrr}
\hline & & & & \\
SGS model & $N_{R}$ & $N_{c d, 0}$ & $\begin{array}{c}\delta_{m} / \delta_{\omega, 0} \\
\text { at } t_{\text {trans }}^{*}\end{array}$ & $\begin{array}{c}t_{\text {peak }}^{*} \\
\text { at } t_{\text {peak }}^{*}\end{array}$ & CPU-hours \\
SMD & 1 & 7484075 & 2.233 & 95 & 2.292 & 264 \\
SMD & 8 & 935509 & 2.230 & 95 & 2.291 & 37 \\
SMD & 16 & 467754 & 2.230 & 95 & 2.287 & 21 \\
SMD & 32 & 233877 & 2.229 & 95 & 2.282 & 14 \\
SMD & 64 & 116938 & 2.227 & 100 & 2.277 & 10 \\
GRD & 1 & 7484075 & 2.358 & 105 & 2.358 & 265 \\
GRD & 8 & 935509 & 2.313 & 105 & 2.317 & 37 \\
GRD & 16 & 467754 & 2.293 & 105 & 2.293 & 21 \\
GRD & 32 & 233877 & 2.277 & 105 & 2.278 & 13 \\
GRD & 64 & 116938 & 2.301 & 105 & 2.302 & 10 \\
SSC & 1 & 7484075 & 2.185 & 105 & 2.189 & 256 \\
SSC & 8 & 935509 & 2.152 & 105 & 2.158 & 38 \\
SSC & 16 & 467754 & 2.135 & 105 & 2.138 & 22 \\
SSC & 32 & 233877 & 2.164 & 105 & 2.166 & 15 \\
SSC & 64 & 116938 & 2.165 & 105 & 2.165 & 11
\end{tabular}

TABLE 7. As table 6 but for LES runs corresponding to DNS case TP600a5.

decreased, but not proportionally since the number of grid points is kept constant; the grid coarsening alone $\left(N_{R}=1\right)$ accounts for a twenty-fold decrease in CPU time for TP600a2 and a ten-fold decrease for TP600a5. An initial eight-fold decrease in the number of tracked drops $\left(N_{R}=8\right)$ leads to an additional five- to seven-fold decrease in CPU time, with greater savings for TP600a5 which has the larger initial number of drops. A further decrease in drops by the same factor (to $N_{R}=64$ ) yields only a two- to four-fold decrement in CPU time, indicating that the CPU-time benefit of increasing $N_{R}$ decreases as $N_{R}$ becomes larger.

Following the format of $\S 5$, we will evaluate the performance of the models by presenting the evolution of the global quantities, followed by flow visualizations, energy spectra, homogeneous-plane statistics and global correlations. The focus is here particularly on TP600a5, because it was not part of the a priori study in Part 1; unless otherwise noted, results for TP600a2 are similar. Results will be presented mostly for $N_{R}=8$, with some comments regarding the influence of the $N_{R}$ value. For analysis purposes, $N_{R}=8$ is the lowest value studied that is greater than unity $\left(N_{R}=1\right.$ uses physical not computational drops), at still considerable computational savings over $N_{R}=1$ and, if accurate, the $N_{R}=8$ LES would demonstrate the feasibility of the computational-drop model.

\subsection{Evolution of the global quantities}

Depicted in figure 9 is the evolution of the global quantities for case TP600a5 with $N_{R}=8$, for LES utilizing the SSC, GRD and SMD models. For all three SGS models, all layers roll up and pair twice. The growth of the layers, as measured by $\delta_{m}(3.1)$, is the same on the DNS and FC-DNS fields. In figure $9(a)$, the SMD-model $\delta_{m}$ is less than the FC-DNS value throughout the layer evolution, and, except for a short period after the second pairing, is also always smaller than the GRD value. Both the GRD and the SSC models slightly over-predict $\delta_{m}$ until the second pairing time, after which the layer growth is under-predicted, with the deviation being greater for the SSC model (that is, the SSC value is larger than the GRD one at earlier times and smaller at later times). Similar results are obtained for TP600a2, except that the SMD $\delta_{m}$ is larger than the GRD value for a longer period after the second pairing, and 

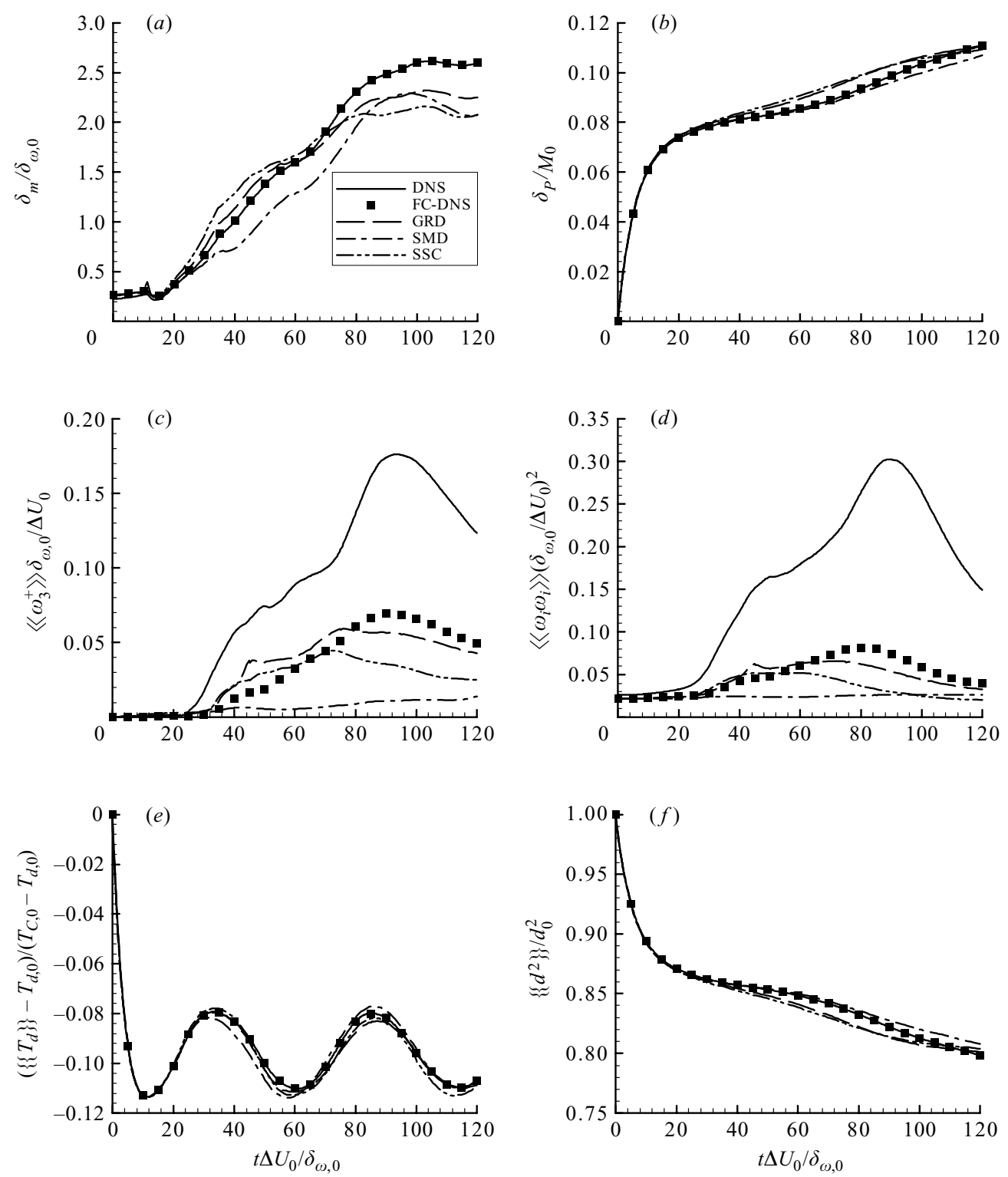

FIGURE 9. Time evolution of non-dimensional global quantities for TP600a5: (a) momentum thickness, $(b)$ product thickness, $(c)$ average positive spanwise vorticity, $(d)$ average enstrophy, $(e)$ average drop temperature and $(f)$ average drop diameter-squared. DNS; FC-DNS with $N_{R}=8$; LES using SMD, GRD and SSC SGS-flux models with $N_{R}=8 . M_{0}=6.214\left(10^{-3}\right) \mathrm{kg}$ is the initial mass in the domain, $d_{0}=7.880\left(10^{-5}\right) \mathrm{m}$.

the SSC $\delta_{m}$ is consistently greater than the GRD one. For TP600a2, compared to the FC-DNS $\delta_{m}$ value of $2.627, \delta_{m}$ at the DNS transition time (see $N_{R}=8$ runs in table 6) is $15 \%, 20 \%$ and $14 \%$ lower for the SMD, GRD and SSC models, respectively. The comparable results for TP600a5 (see $N_{R}=8$ runs in table 7) are $15 \%, 11 \%$ and $18 \%$ lower for the SMD, GRD and SSC models, respectively. These $\delta_{m}$ errors are larger than those obtained for SP600 LES. Similar to SP600, the peak momentum thickness time $t_{\text {peak }}^{*}$ for the TP600a2 LES, listed in table 6, occurs sooner and has a smaller $\delta_{m}$ 
than the FC-DNS. For TP600a5 (table 7), $t_{\text {peak }}^{*}$ is well-predicted by the SSC and GRD models but occurs sooner for the SMD model; however its $\delta_{m}$ is under-predicted by all models. For both TP600a 2 and TP600a5, $\delta_{m}$ is minimally affected by the value of $N_{R}$ (see tables 6 and 7 ).

The global mixing of the TP600a5 layers, as measured by the product thickness $\delta_{P}=\int_{\text {domain }} \rho\left[2 \min \left(Y_{V}, Y_{C}\right)\right] \mathrm{d} V$ in figure $9(b)$, is the same on the DNS and FC-DNS fields; $\delta_{P}$ is predicted best by the SMD model. The GRD prediction is also very close to the FC-DNS, with a slight over-prediction from the GRD model between the first pairing and the DNS transition time; the SSC model leads to similar results. The same trends are found for TP600a2, although the SMD $\delta_{P}$ curve is much closer to the FC-DNS. Although the SMD and GRD models seem comparable when considering $\delta_{m}$ and $\delta_{P}$, dramatic differences emerge when the vorticity activity of the layers is examined. The global positive spanwise vorticity $\left(\left\langle\left\langle\omega_{3}^{+}\right\rangle\right\rangle\right.$in figure $\left.9 c\right)$ and enstrophy $\left(\left\langle\left\langle\omega_{i} \omega_{i}\right\rangle\right\rangle\right.$ in figure $\left.9 d\right)$ display similar behaviour as for SP600 (figure 4), with the GRD model yielding good predictions while the SMD model exhibits strong departures from the FC-DNS and minimal vorticity activity. The SSC predictions are somewhat inferior to those obtained with the GRD model in that the vorticity activity is further reduced from the FC-DNS.

Finally, the drop-ensemble-average (denoted $\{\{\}\}$ ) of temperature $T_{d}$ and diametersquared $d^{2}$ (figures $9 e$ and $9 f$ for TP600a5), of the FC-DNS drop field, which match those of the DNS (physical) drop field, are closely followed by all three LES models. For $\left\{\left\{T_{d}\right\}\right\}$, the GRD model overlaps the FC-DNS until $t^{*} \simeq 45$, approximately the time of the first pairing, after which it is smaller until $t^{*} \simeq 95$, while the SMD slightly under-predicts $\left\{\left\{T_{d}\right\}\right\}$ until $t^{*} \simeq 65$, and thereafter slightly over-predicts it until $t^{*} \simeq 95$; at the later times, after $t^{*} \simeq 95$, both the GRD and SMD predictions overlap the FCDNS. The SSC model tends to a minor over(under)-prediction of $\left\{\left\{T_{d}\right\}\right\}$ before(after) $t^{*} \simeq 45$. As elaborated by Le Clercq \& Bellan (2004), the oscillations in $\left\{\left\{T_{d}\right\}\right\}$ are due to the competing effects of heating, which removes energy from the gas phase, and evaporation, which releases energy into the surrounding gas. In considering $\left\{\left\{d^{2}\right\}\right\}$, it is apparent that, because this is an ensemble-average result, the drops do not follow the single-drop ' $d^{2}$ law' (linear decay; Williams 1965); after an initial period of high rate of $\left\{\left\{d^{2}\right\}\right\}$ decrease, this rate subsides as the increasing $Y_{V}$ impedes evaporation. The SMD model is slightly superior to the other two models, which have virtually identical results for $\left\{\left\{d^{2}\right\}\right\}$. The three SGS-flux models display slightly better predictions of $\left\{\left\{T_{d}\right\}\right\}$ and slightly worse predictions of $\left\{\left\{d^{2}\right\}\right\}$ for TP600a5 than for TP600a2.

The global quantities show that $\delta_{m}, \delta_{P},\left\{\left\{T_{d}\right\}\right\}$ and $\left\{\left\{d^{2}\right\}\right\}$ are slightly better predicted by the SMD model than by the SSC and GRD models. However, this superiority of the SMD model is strongly negated by its poor predictions of the SRS activity, for both the positive spanwise vorticity and the enstrophy. Furthermore, as shown below, despite having good predictions for $\delta_{P},\left\{\left\{T_{d}\right\}\right\}$ and $\left\{\left\{d^{2}\right\}\right\}$, the SMD model fails to capture the details of the spatial distribution of the drops.

\subsection{Flow visualizations}

Flow visualizations allow a more comprehensive qualitative comparison of the LES to the FC-DNS; all plots presented are for TP600a5 at the DNS transition time $t_{\text {trans }}^{*}=105$. The spanwise and streamwise vorticity are plotted in figure 10 for the FC-DNS and the SMD, GRD and SSC LES (compare to figures 5 and 6 for SP600). Once again, the SMD model is shown to be incapable of generating the SRS structures, while the GRD and SSC models qualitatively capture the FC-DNS vorticity 

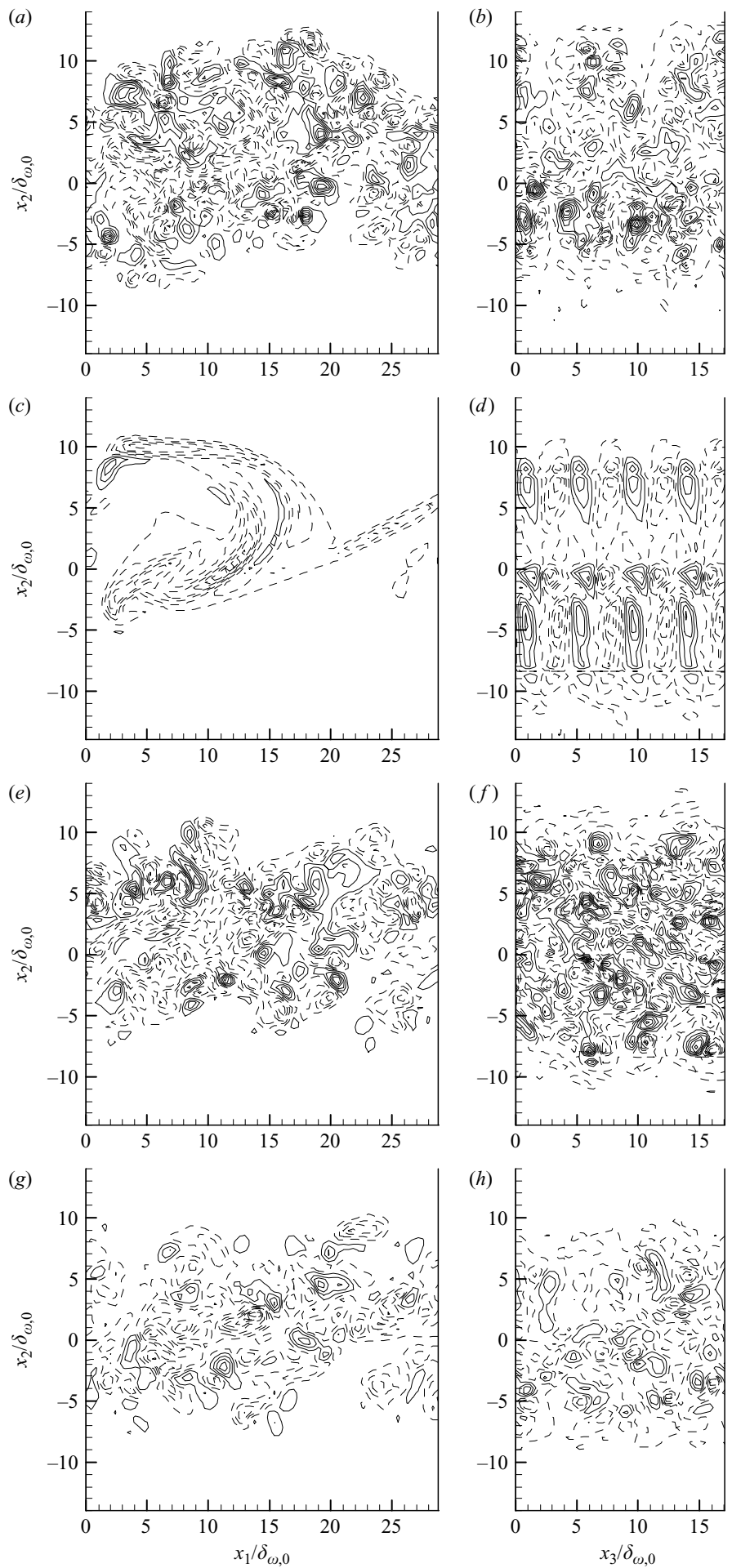

FIGURE 10. Spanwise vorticity (left, $x_{3} / L_{3}=0.5$ ) and streamwise vorticity (right, $x_{1} / L_{1}=0.5$ ) for TP600a5 with $N_{R}=8$, at $t^{*}=105:(a, b)$ FC-DNS and $(c-h)$ LES using $(c, d)$ SMD, $(e, f) \mathrm{GRD}$ and $(g, h) \mathrm{SSC}$ SGS-flux model. Contour levels range from -0.818 to 0.499 in increments of 0.094 for $\omega_{3} \delta_{\omega, 0} / \Delta U_{0}$ and from -0.670 to 0.891 in increments of 0.112 for $\omega_{1} \delta_{\omega, 0} / \Delta U_{0}$ (15 levels for both). 
distribution. However, while the SRS activity is qualitatively well duplicated by the SSC and GRD models, what is clearly under-predicted is the size of the large spanwise vortex; this is totally consistent with figure $9(a)$ which shows the under-prediction of $\delta_{m}$ by both these models. The streamwise vorticity is similar to the SP600 result in figure 6 in that for the GRD and SSC models it mimics that of the FC-DNS, while for the SMD model it retains the structure of the initial condition perturbations.

The cross-stream mixing of the vapour and drops can be assessed from analysis of figure 11, which shows the TP600a5 vapour mass fraction $\left(Y_{V}\right)$ and drop number density $\left(\rho_{n}=N_{\beta} / V_{f}\right.$, where $N_{\beta}$ is the number of drops in the filtering volume $V_{f}$ ), for the FC-DNS and the SMD, GRD and SSC LES, all with $N_{R}=8$, in the between-thebraid plane. Equivalent plots for TP600a2 are similar except for lower drop number density. Figure 11(a) shows a complex FC-DNS drop organization, with preferential distribution away from high-vorticity regions into low-vorticity regions depicted in figure 10(a) (also see Part 1). Since figure 11(a) is virtually identical to the equivalent figure for the FC-DNS $\rho_{n}$ computed with the full physical drop field $\left(N_{R}=1\right.$; not shown), $N_{R}=8$ does accurately capture the liquid-phase situation. Inside the layer, regions of high $\rho_{n}$ correspond to the highest $Y_{V}$ regions, portrayed in figure $11(b)$. The highest $Y_{V}$ overall occurs in the lower stream, which has a nearly uniform drop distribution at a value intermediate in the $\rho_{n}$ range. The FC-DNS $Y_{V}$ plots show the ultimate vortex as the largest structure, with complex SRS activity within it. In considering the SMD model prediction, both $\rho_{n}$ (figure 11c) and $Y_{V}$ (figure $11 d$ ) show a qualitatively different aspect than the FC-DNS. The SMD model has captured the ultimate vortex, but it is devoid of any SRS activity; the drops are mostly concentrated at the vortex edges with few drops within the layer, the highest drop concentration occurring in a narrow band in the lower stream around the ultimate vortex. Therefore, the indications are that the SMD model is an inadequate SGS model, since it fails in its primary purpose of reproducing the SRS motions of the flow. In contrast, the GRD model has predictions that are both qualitatively correct and quantitatively close to the FC-DNS field, in that the $\rho_{n}$ distribution (figure 11e) follows the FC-DNS in both the lower-stream distribution as well as the regions of high $\rho_{n}$ within the layer. The GRD $Y_{V}$ field (figure $11 f$ ) shows both large-scale and SRS activity, although, as expected, smeared due to the solution being obtained on a coarser (LES) grid. The SSC model predictions (figure $11 \mathrm{~g}$ and $11 \mathrm{~h}$ ) are also qualitatively similar to the FC-DNS, although the mass fraction in the lower part of the mixing region is slightly smaller.

Therefore, from figure 11, the gas-phase SGS-flux model is here seen to substantially affect the spatial distribution of the drops. Because, in the present LES approach, the computational drops obey the same equations as physical drops, they will likewise be preferentially distributed into low-vorticity regions. Whether the final drop distribution mimics the FC-DNS depends on whether the SGS models are able to create the physically correct low-vorticity regions. As demonstrated by the comparison just discussed, the GRD and SSC models are successful in capturing the distribution of drops observed in the FC-DNS.

To ascertain the effect of computational-drop modelling, illustrated in figure 12 are TP600a5 GRD LES results obtained with sequentially smaller numbers of computational drops. Notably, comparing the $N_{R}=8$ result (previously depicted in figures $11 e$ and $11 f$ ) to $N_{R}=1$ (figures $12 a$ and $12 b$ ), no new qualitative features are observed in either the $\rho_{n}$ or the $Y_{V}$ plots when the number of computational drops is reduced from the number of physical drops, although for $N_{R}=1$ the drop distribution in the lower stream is more uniform. The $N_{R}=16$ LES (figures $12 c$ 

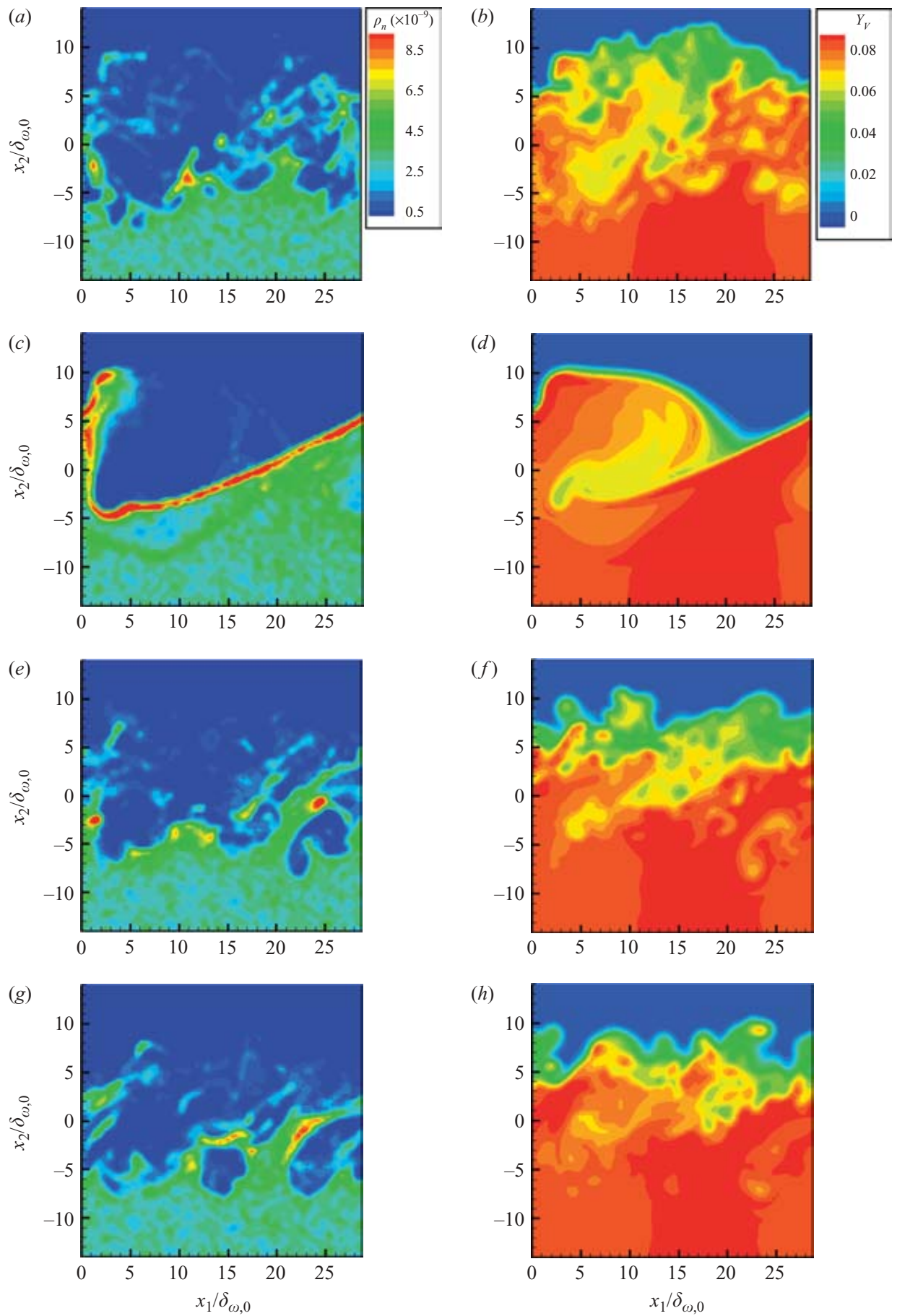

FIGURE 11. Drop number density in $\mathrm{m}^{-3}$ (left) and vapor mass fraction (right) for TP600a5 with $N_{R}=8$, at $t^{*}=105$ in the between-the-braid plane $\left(x_{3} / L_{3}=0.5\right):(a, b)$ FC-DNS; $(c-h)$ LES using $(c, d) \mathrm{SMD},(e, f)$ GRD and $(g, h)$ SSC SGS-flux model. 


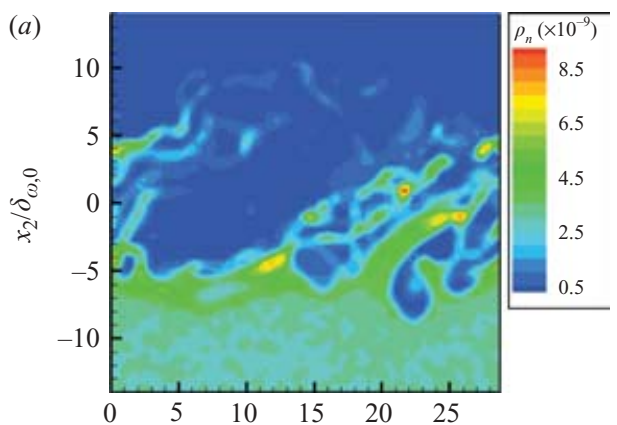

(b)
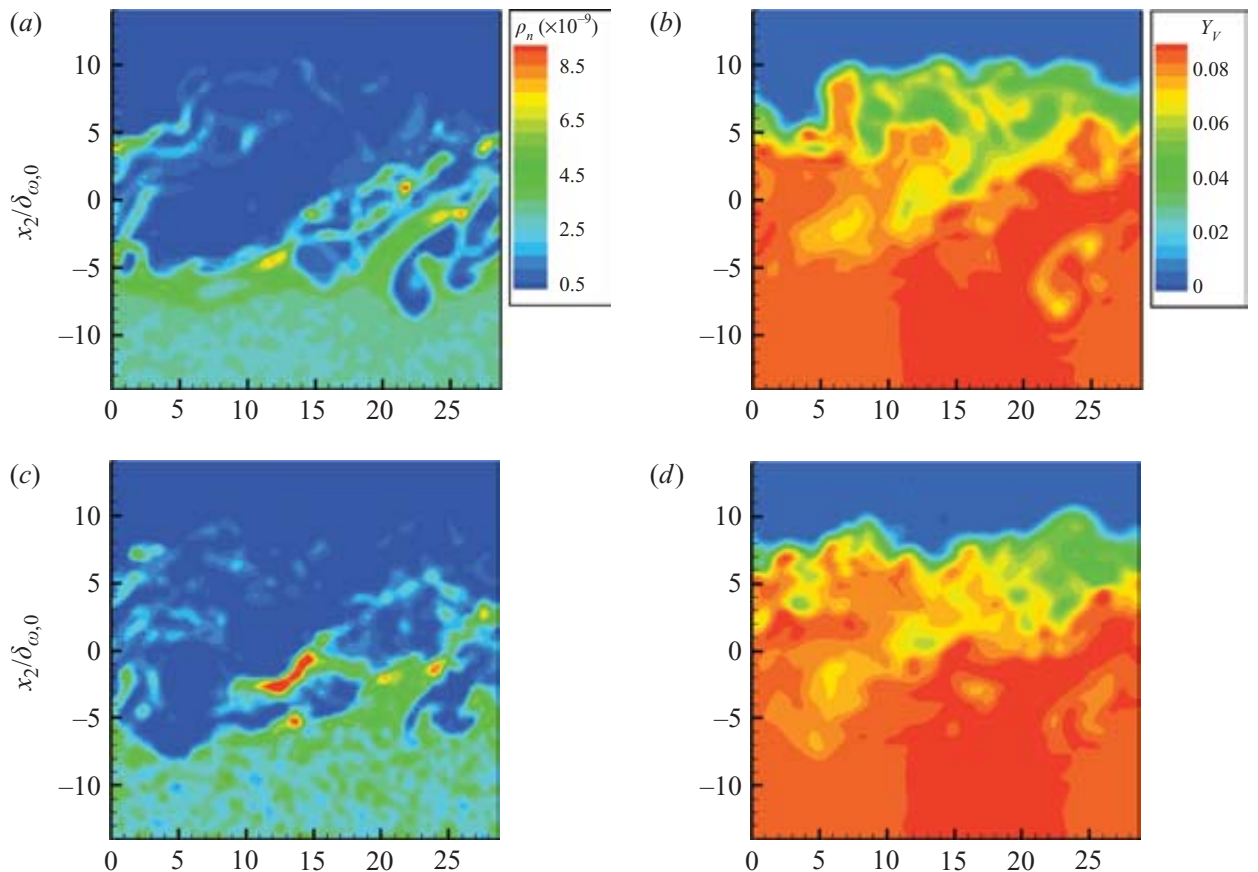

(d)
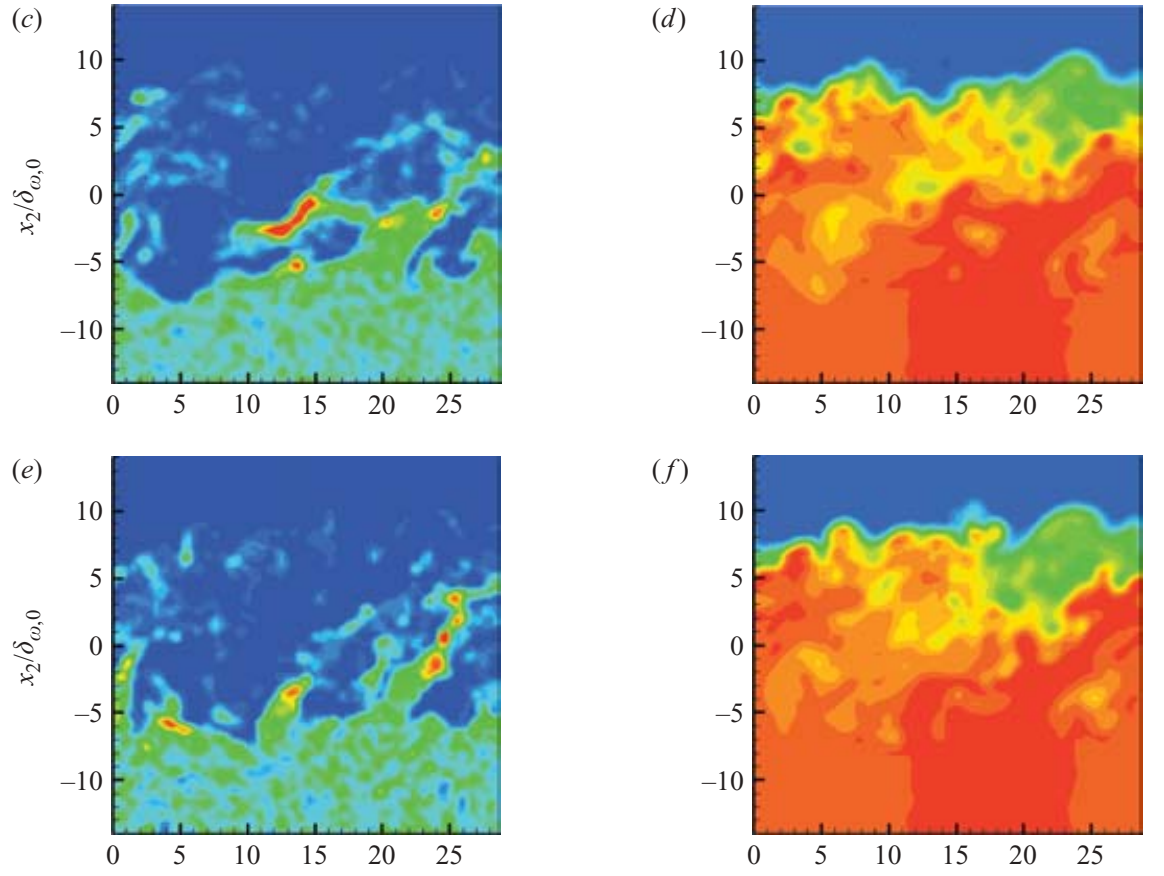

$(f)$
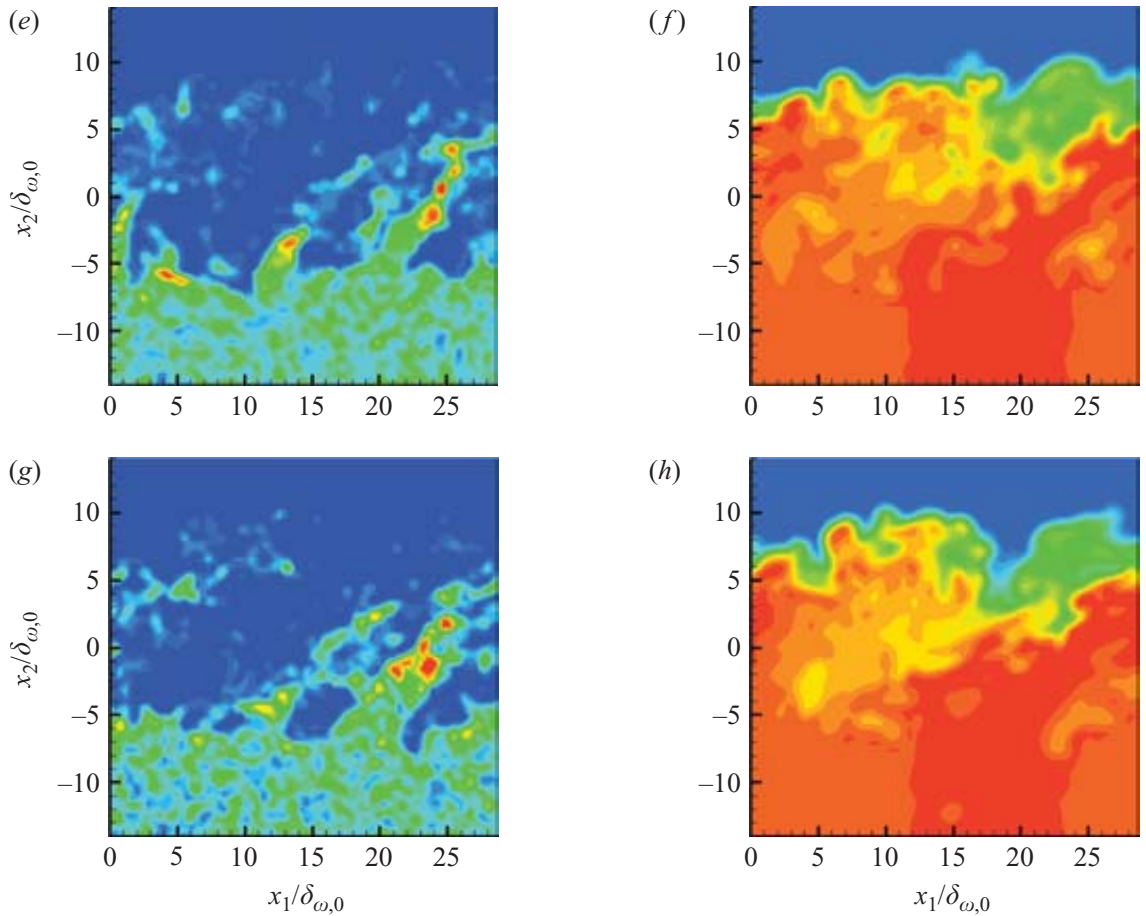

(h)

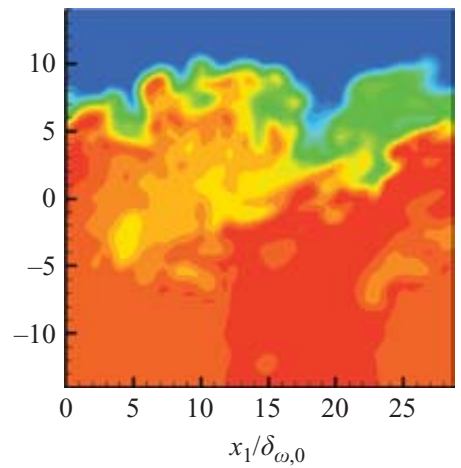

FIGURE 12. Drop number density in $\mathrm{m}^{-3}$ (left) and vapor mass fraction (right) for TP600a5 LES using the GRD model, at $t^{*}=105$, in the between-the-braid plane $\left(x_{3} / L_{3}=0.5\right)$ : $(a, b) N_{R}=1,(c, d) N_{R}=16,(e, f) N_{R}=32$ and $(g, h) N_{R}=64$. 

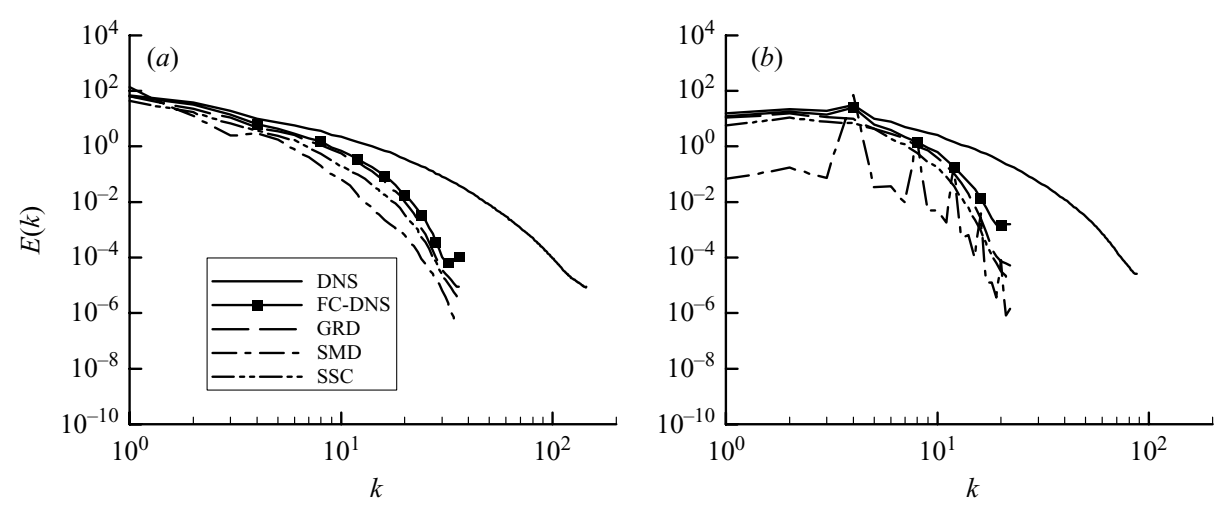

FIGURE 13. Energy spectra of $u_{1}$ for TP600a5 at $t^{*}=105:(a)$ streamwise and $(b)$ spanwise. Energy in $\mathrm{m}^{2} \mathrm{~s}^{-2}$.

and $12 d)$ is virtually the same as the $N_{R}=8$ result. The accuracy of the GRD model shows no significant degradation when $N_{R}$ is increased to 32 (figures $12 e$ and $12 f$ ), although the lower-stream $\rho_{n}$ distribution is less uniform. Finally, for $N_{R}=64$, the aspect of the layer is still qualitatively correct for the vapor mass fraction (figure $12 h$ ). For TP600a2 with $N_{R}=64$, regions of very low and very high drop number density appear in the lower stream, which do not appear at smaller $N_{R}$. Therefore, from these simulations, the indications are that the maximum drop reduction that replicates both the drop field and the fuel vapour should be $N_{R}=32$; the drop reduction by a factor of 32 from the DNS is less than the 64-fold reduction in the number of grid points.

\subsection{One-dimensional spectra of velocity components}

To assess the energy content of various scales of the flow, one-dimensional streamwise and spanwise energy spectra were computed for both TP600a2 and TP600a5; these spectra resemble those of SP600 (figure 7). Figure 13 clearly shows the energy versus wavenumber $(k)$ distribution of the GRD model to be the closest to the FC-DNS, with the SSC closely following the GRD results, while the SMD model is the farthest from the FC-DNS. For the spanwise spectra, sharp peaks are evident for the SMD model, corresponding to the structures seen in the streamwise vorticity plots (in figure 10), which result from persistence of the initial perturbation. In contrast, the SSC and GRD models show no such structures. Therefore the same conclusions as distilled from the SP600 results hold: the SMD model does not allow the development of true turbulence.

\subsection{Homogeneous-plane gas-phase statistics}

Selected homogeneous-plane averages and fluctuations are presented in figure 14 for TP600a5, for comparison with figure 8 for SP600 (being null for SP600, $Y_{V}$ was not plotted there). Due to the temperature difference between drops and carrier gas, and the ensuing evaporation, the thermodynamic quantities differ between the two free streams. The DNS and FC-DNS overlap for the planar averages of all plotted quantities; whereas for SP600 the $T$ fluctuations overlapped for DNS and FC-DNS while the $T$ averages differed, for TP600a5 the opposite behaviour is observed. As noted previously, the difference between the DNS and the FC-DNS quantities indicates sensitivity to the SGSs that are lacking in the FC-DNS. In predicting the averages and fluctuations of $u_{1}$ and $u_{3}$ (not shown), all models perform much as they did for SP600, with comparably good predictions for $u_{1}$, and the SMD somewhat 

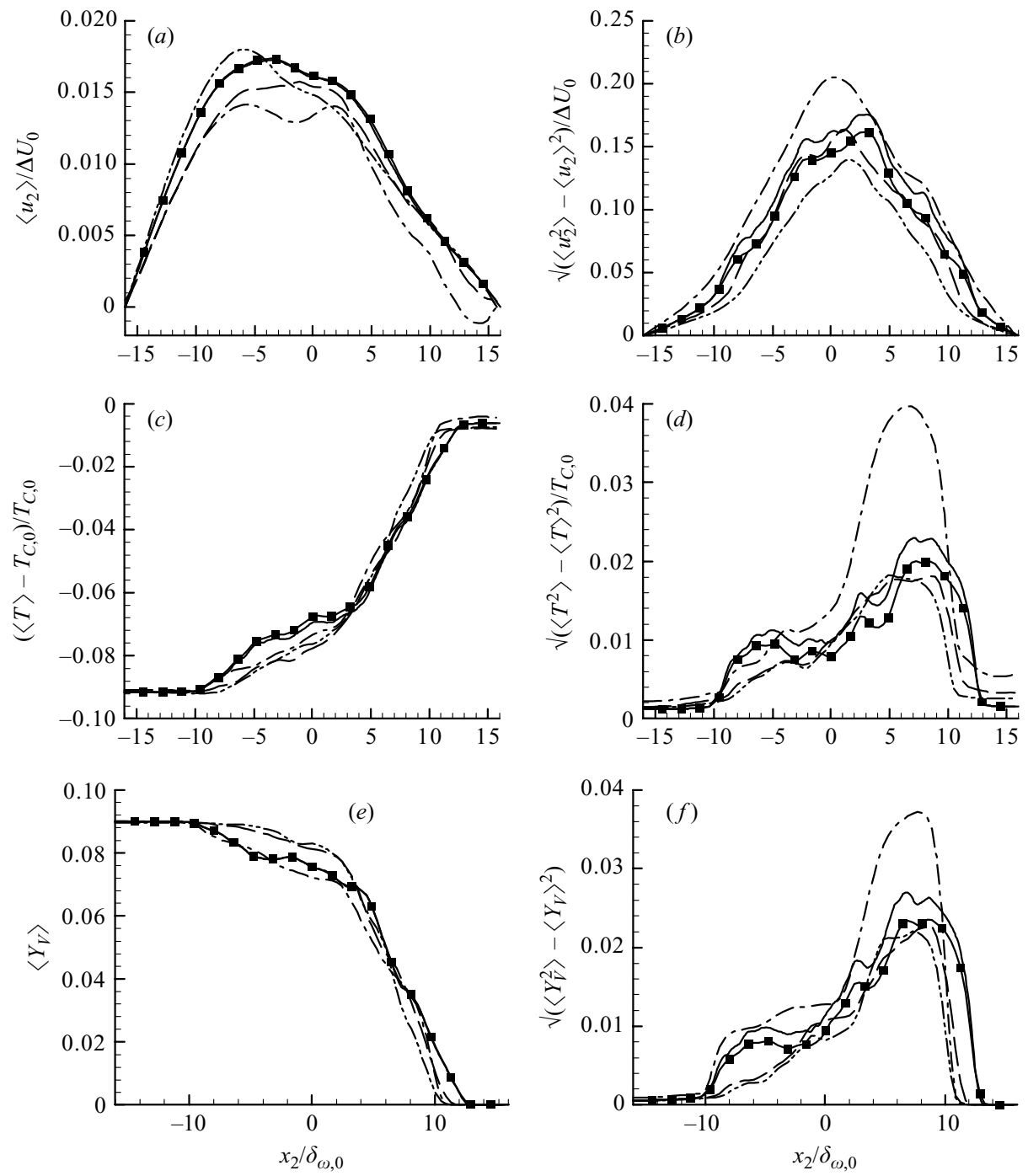

Figure 14. Homogenous-plane statistics of gas-phase quantities for TP600a5 at $t^{*}=105:(a, b)$ $u_{2},(c, d) T$ and $(e, f) Y_{V}$. See figure 13 for legend. Averages (left) and fluctuations (right).

worse than the other models, under-predicting the $u_{3}$ fluctuations even more. However, noticeable improvement is observed in predicting $u_{2}$ averages, for which all models yield reasonable results, indicating that the different $u_{2}$ average profile is easier to predict in the TP600a5 case. On the other hand, for the SMD and SSC models, the comparison with FC-DNS for the $u_{2}$ fluctuations is less favourable in the TP600a5 case. For both averages and fluctuations of $T$ and $Y_{V}$, the GRD and SSC results are similar; both models give good results in the upper stream, but have some overor under-predictions in the lower stream. The SMD performs similarly to the other two models in predicting the $T$ averages. The SMD model is the best for the $Y_{V}$ averages, and for the lower-stream fluctuations of $T$ and $Y_{V}$, but is the worst for the upper-stream fluctuations of $T$ and $Y_{V}$. Regarding $\rho$ and $p$ (not shown), the averages are predicted better by all models for TP LES than for SP LES, with the SMD 

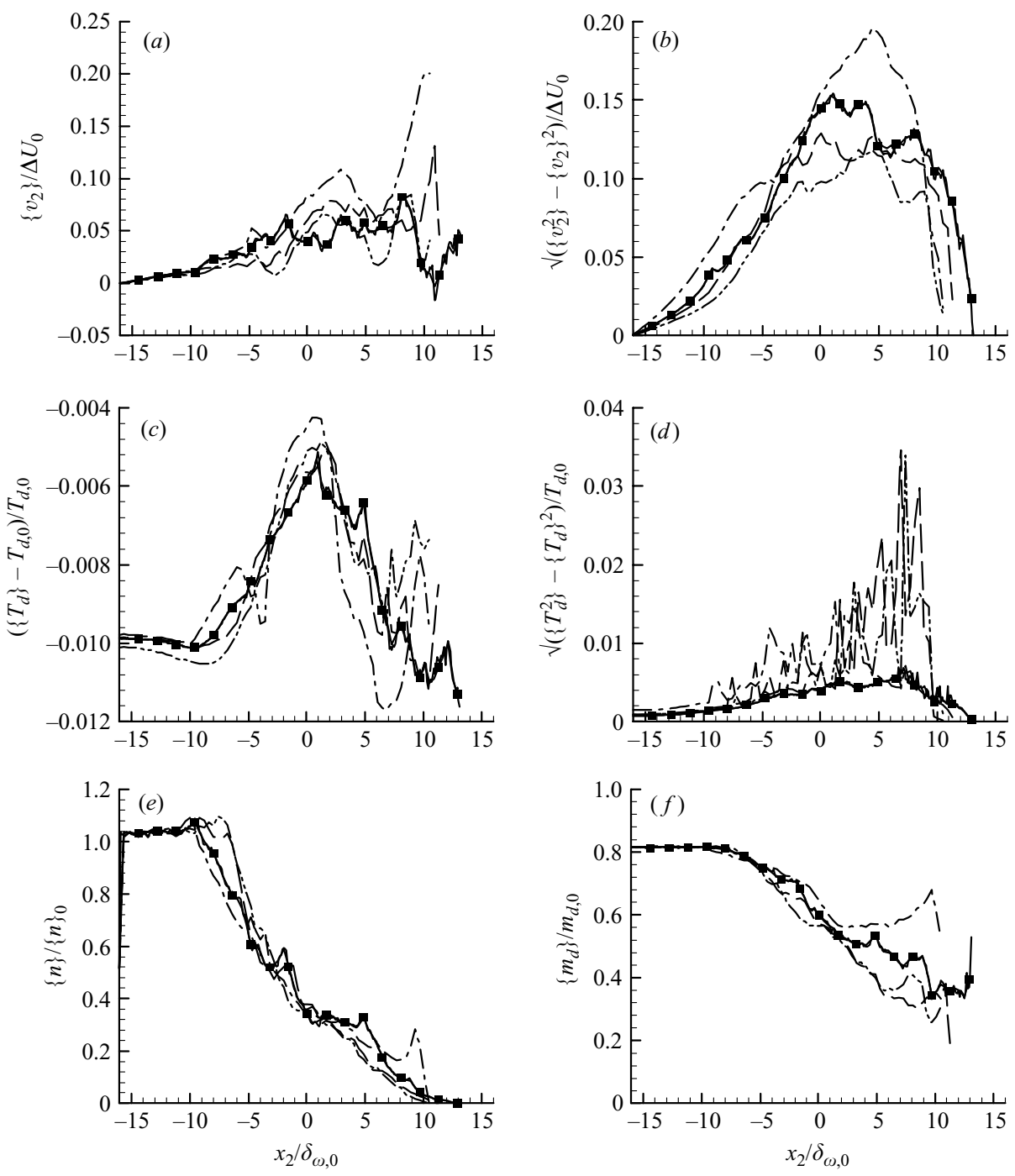

FIGURE 15. Homogeneous-plane statistics of drop-phase quantities for TP600a5 at $t^{*}=105$ : $(a, b) v_{2},(c, d) T_{d}$, (e) number of drops, $n,(f) m_{d} \cdot m_{d, 0}=1.645\left(10^{-10}\right) \mathrm{kg},\{n\}_{0}$ is the initial plane-average number of physical drops: 47829 for DNS, 191349 for FC-DNS and LES. See figure 13 for legend. Averages $(a, c, e, f)$ and fluctuations $(b, d)$.

showing the greatest inaccuracy in duplicating the $\rho$ and $p$ fluctuations. Therefore, the SMD model is inferior to the GRD and SSC model only when the upper-stream thermodynamic fluctuations are considered, but is comparable to them for the other planar quantities. Similar to the discussion for SP600, these planar results need to be cautiously interpreted, since they do not reveal the qualitative differences among the models that were observed in the contour plots in figures 10 and 11.

\subsection{Homogeneous-plane liquid-phase statistics}

Parallel to the gas-phase statistics just discussed, drop-based planar averages and fluctuations for TP600a5 LES are plotted in figure 15 for the drop velocity, mass and temperature. For the drop-phase statistics, the value of each dependent variable at a 
grid point $x_{2, j}$ is computed using all the drops located in $x_{2, j}-\Delta x_{2} / 2<X_{2}<x_{2, j}+$ $\Delta x_{2} / 2$ (where $X_{2}$ and $\Delta x_{2}$ are the drop position and the grid spacing, respectively, in the cross-stream direction); the average over these drops is denoted \{\} and is undefined in the portion of the domain without drops. Some of the drop-based statistics, especially for $T_{d}$, suffer from the sparsity of drops in the upper stream. Figure 15 also includes the average number of physical drops, $\{n\}$, predicted by all models, which is comparable (figure 15e), as the planar averaging generally masks the obvious differences in drop spatial distribution that were observed in figure 11. For the SMD case, peaks in the upper-layer region at the edge of the free stream indicate that the drops congregate at incorrect locations, that is, in regions other than those of the FC-DNS.

The performance of the models in predicting the drop velocity statistics mimics that for the gas-phase velocity (only $v_{2}$ is shown): the average of $v_{1}$ is equally well predicted by all models; overall the SSC model gives the best results for $v_{3}$ averages; the GRD model tends to over-predict the $v_{3}$ average, while the SMD model tends to underpredict it; for the velocity fluctuations, the GRD and SSC models generally perform better than the SMD model, whose $v_{3}$ fluctuations inside the layer are less than half of the FC-DNS fluctuations. The average mass of the drops (figure $15 f$ ) is well-predicted by all LES in the lower stream. In the upper part of the layer, the $m_{d}$ average is somewhat under-predicted by both the SSC and GRD models, yet is nonetheless closer to the FC-DNS than is the SMD average; the large discrepancies between the SMD case and the FC-DNS are consistent with the peak identified in figure $15(e)$ in the same region. All models have reasonable predictions for the $m_{d}$ fluctuations (not shown). The three models give distinctly different results for the $T_{d}$ average (figure $15 c$ ) with the GRD model giving good agreement with the FC-DNS throughout the layer. The SSC $T_{d}$ averages are similar to the GRD, except for under-prediction in the lower stream. For the SMD model, the $T_{d}$ average exhibits a physically incorrect nonmonotonic behaviour in the lower part of the layer; moreover, the SMD-model drops are too cold in the upper part of the layer. While the SMD model is the worst for the $T_{d}$ averages, it has the best $T_{d}$ fluctuations (figure $15 d$ ). In the upper stream, the SMD model agrees well with the FC-DNS for the $T_{d}$ fluctuations, whereas the SSC and GRD models greatly over-predict them; in the lower stream, all models over-predict the $T_{d}$ fluctuations. The error in $T_{d}$ is here conjectured to result from its sensitivity to the gas-phase flow field, which in LES is interpolated to the drop locations with a larger grid spacing than in DNS; therefore, this error may be inherent in LES.

\subsection{Flow-field correlations}

Finally, the LES using the SSC, SMD and GRD models with various values of $N_{R}$ were correlated with the FC-DNS using least-squares fits (3.3); the results yield similar values to those for SP600 (see $\S 5.5$ and table 5) for all SP variables. Comparing the SSC, SMD and GRD models, the slopes and correlations, which ideally would be unity, are similar, although those for the SMD model are generally the smallest and those of the GRD model are generally the largest. For $Y_{V}$ (which was null for SP600), the slopes were in the range 0.95 to 0.97 across the SGS models and $M L_{0}$ values, and the correlations ranged from 0.86 to 0.94 with the best being for the GRD and the worse for SMD. Therefore, as for SP flow, comparisons of the LES flow field to DNS data must be made from a different standpoint than global correlations. These results were independent of $N_{R}$, even when $N_{R}$ was increased to 64. Thus, it appears that global correlations, if used to compare simulations with experimental data (here represented by the FC-DNS), may not be able to identify a model deterioration when 


\begin{tabular}{|c|c|c|c|c|c|c|c|c|c|c|c|}
\hline \multicolumn{6}{|c|}{ Slopes } & \multicolumn{6}{|c|}{ Correlations } \\
\hline$N_{R}$ & 1 & 8 & 16 & 32 & 64 & $N_{R}$ & 1 & 8 & 16 & 32 & 64 \\
\hline $\bar{S}_{I}$ & 1.23 & 1.34 & 1.40 & 1.32 & 1.34 & $\bar{S}_{I}$ & 0.71 & 0.72 & 0.72 & 0.69 & 0.69 \\
\hline $\bar{S}_{I I, 1}$ & 0.89 & 0.81 & 0.83 & 0.77 & 0.75 & $\bar{S}_{I I, 1}$ & 0.59 & 0.58 & 0.57 & 0.55 & 0.54 \\
\hline $\bar{S}_{I I, 2}$ & 0.55 & 0.55 & 0.53 & 0.57 & 0.52 & $\bar{S}_{I I, 2}$ & 0.49 & 0.46 & 0.43 & 0.47 & 0.42 \\
\hline $\bar{S}_{I I, 3}$ & -0.05 & -0.01 & -0.02 & 0.05 & 0.02 & $\bar{S}_{I I, 3}$ & -0.03 & 0.00 & -0.01 & 0.03 & 0.01 \\
\hline $\bar{S}_{I I I}$ & 1.25 & 1.36 & 1.43 & 1.35 & 1.36 & $\bar{S}_{I I I}$ & 0.68 & 0.69 & 0.69 & 0.65 & 0.66 \\
\hline
\end{tabular}

TABLE 8. Slopes (exact/model) and correlations from least-squares fit of FC-DNS to LES flow-field quantities for LES runs in table 7 using GRD Model: TP600a5 at $t^{*}=105$.

$N_{R}$ is increased. Therefore, at this point, flow visualizations appear to be the best diagnostic for selecting $N_{R}$.

Similar slopes and correlations for the FSTs were tabulated for the SSC, SMD and GRD models with various values of $N_{R}$; as an example, the results for TP600a5 using the GRD model are presented in table 8. In contrast to the a priori analysis (Part 1 and $\S 4$ above), where the FSTs were always over-predicted, for the SSC-model and GRD-model LES, some FSTs are instead under-predicted. For the SMD-model LES, the FSTs are over-predicted, but the slopes are not necessarily farther from unity than those from the GRD model; however, the correlations are generally lower. The SSC model has similar correlations as the GRD model. Similar to the findings for the flow field, there appears to be little impact on the correlations upon increasing $N_{R}$ from 1 to 64 .

\subsection{Summary of TP LES results}

The TP LES have generally confirmed the conclusions reached from the SP LES regarding the SGS-flux modelling. From the standpoint of numerical stability, it appears that the TP LES is more sensitive to the SGS-flux model than is the SP LES; specifically, the GRC model is unstable despite using the DNS-calibrated coefficients that were a priori statistically equivalent for TP and SP flows. However, when numerically stable, the SGS-flux models performed similarly for SP and TP LES; in particular, the SMD model showed in both cases an inability to generate SRSs while the GRD and SSC models led to flow fields with SRS structures that mimicked the FC-DNS. For the GRD and SSC models, the spatial distribution of drop number density and of vapour that had emanated from the drops was consistent with the FC-DNS, and the LES predictions were also quantitatively good. For the SMD model, the poor replication of the SGS activity led to a distinctively incorrect drop spatial distribution, with drops concentrated at the incorrect locations. Thus, the benefit of having compared the SGS-flux models in the SP flow is evident, in that the inadequacies of the SMD LES can incontrovertibly be attributed to the gas phase; more generally, SGS-flux models that perform poorly for SP flows can be eliminated in advance from consideration for TP flows. LES conducted with a 64-fold reduction in the number of drops compared to the DNS showed that the SSC-model and GRD-model LES exhibited good results, although with slightly diminished accuracy compared to a 32-fold reduction in the number of drops. Further increases in the number of computational drops did not significantly improve the results, although the analysis focused on the LES with an eight-fold reduction in drops, which still achieved considerable computational savings over the DNS. 


\section{Conclusions}

Large-eddy simulation (LES) has been performed of three-dimensional temporal mixing layers, both single-phase (SP) and two-phase (TP) with evaporating drops. The LES results have been compared to previous direct numerical simulation (DNS), documented in Part 1, conducted at the same initial vorticity-thickness Reynolds number (600) and mass loading conditions. Both DNS and LES used an Eulerian frame for the gas phase and a Lagrangian frame for the drop (liquid) phase. The coupling between the phases is provided by source terms in the gas-phase equations and by each drop having as its far field the gas-phase variables at its location. In order to create a consistent LES methodology, in which both the number of grid points and tracked drops can be reduced compared to the DNS, we have used the LES methodology developed in Part 1 of this study. The LES follows the DNS as much as possible, and requires models only for the subgrid-scale (SGS) fluxes and filtered source terms (FSTs) in the gas-phase equations. Computational-drop modelling is used in LES such that the evolution of the computational drops is governed by the same equations as govern the physical drops; then each computational drop is taken to represent a (fixed) number of physical drops. The unfiltered flow field required for the calculation of each drop's source-term contribution is approximated by the filtered flow field. Both DNS and LES are computed using eighth-order finite differences, fourth-order Runge-Kutta temporal integration and fourth-order interpolation of gas-phase variables from grid points to drop locations; maintaining the same numerical scheme removes the possibility of numerically added dissipation in LES when compared to DNS. All LES were conducted with a grid spacing that was four times larger than the DNS (64-fold reduction in the number of grid points).

Since the a priori study of the SGS-flux models (Part 1) showed statistical equivalence of model coefficients for transitional states of SP and TP flows, the SP LES were undertaken to independently study SGS-flux modelling without FST modelling. For the SP layer, we have tested constant-coefficient SGS-flux models, which were previously calibrated on the DNS database (Part 1), and dynamic SGS-flux models. Both the constant-coefficient and dynamic Smagorinsky models (SMC and SMD, respectively) were numerically stable but led to LES flow fields unlike the filtered-andcoarsened (FC) DNS flow fields in that they were devoid of small-resolved-scale (SRS) activity. While the Smagorinsky model might perform better for flow configurations requiring turbulent initial conditions and/or when solid walls (at which vorticity is always generated) are present, this model is deemed unsuitable for the present mixinglayer situation where the flow is required to undergo transition to turbulence from a laminar initial condition. The other three models considered, constant-coefficient and dynamic gradient models (GRC and GRD respectively) and the scale-similarity model (SSC), led to good predictions for the filtered flow field.

For the TP layer, we conducted LES at two mass loadings, 0.2 and 0.5 . The GRC model was not consistently numerically stable and the SMC model was eliminated due to its poor accuracy for SP flow; thus LES were performed with SSC, GRD and SMD models. The computational-drop and FST modelling performed well at both mass loadings considered. Although by some measures - such as momentum and product thicknesses, drop-averaged temperature and diameter-squared, homogeneous-plane statistics (averages and fluctuations) and flow-field correlations - the three models yielded similar results, the LES flow fields they produced were qualitatively different. The SMD-model LES field lacked SRS activity and had drops concentrated on the edges of the ultimate vortex, while the SSC-model and GRD-model LES resembled the FC-DNS in having significant SRS structures, with drops dispersed throughout the 
ultimate vortex and preferentially concentrated in low-vorticity regions. The behaviour of the models in predicting the gas-phase flow field of TP LES paralleled their performance in the SP LES, and moreover corresponded to the a priori correlation between the models and the SGS fluxes. Measures based on the vorticity, namely average positive spanwise vorticity and enstrophy, were found to correlate well with the prevalence of SRS structures. This led to the conclusion that the measures traditionally used to justify the Smagorinsky model, such as planar quantities, may be misleading, and that the assessment of the capabilities of an LES model should include an examination of the local behaviour of the flow field and drop variables.

By systematically comparing LES that were conducted using different numbers of computational drops, it was possible to discern the number of computational drops required to preserve the qualitative aspects of LES using physical drops. We note that using physical drops in LES yields significant computational savings over DNS due to the grid coarsening alone, but as indicated by the a priori study, the physical drop evolution is not necessarily more accurate than that of computational drops since the flow field encountered by the drops is different in LES than in DNS. A good starting point for determining the number of computational drops was to use the same reduction as for the grid spacing. The determination of the minimum number of computational drops that can yield acceptable accuracy, in the general case where DNS or experimental data are absent, remains an open question.

In essence, we have shown that two-phase LES can be successfully conducted using SGS-flux models developed for SP flows. However, despite statistical a priori equivalence of constant-coefficient models, not all stable models in the SP case were also stable for TP flows, although all those that were stable for TP flows were also stable for SP flows. This difference in stability characteristics may be attributed to the drops, whose source terms were found a priori to be the major contribution of (entropy) dissipation for the gas-phase. Unlike the flux-related dissipation, which is the sole SP dissipation mechanism and is always positive semi-definite, the sourcerelated dissipation may be negative or positive at the SGS, that is, adding or removing entropy from the resolved flow field. Therefore, while SGS-flux models can be derived and tested in computationally less demanding SP flow, they must be verified on TP flows to account for the interaction between the SGS-flux models and drop evolution. Numerical stability was achieved without the use of mixed models (Bardina et al. 1980; Vreman et al. 1997), which contributed to the computational efficiency. The GRD SGS-flux model used in this study was more accurate than the Smagorinsky model, while being no more computationally expensive or algorithmically complicated. The GRD model performed comparably to the SSC model, but unlike it, has the advantage of not requiring an a priori calibrated coefficient. The GRD model has also shown an ability to handle both laminar and transitional flows, a notoriously difficult test. This model must be further tested at higher Reynolds numbers and for spatial configurations, and then be validated with experimental data, in order to determine its suitability for routine LES simulations.

Two main areas of model development are therefore anticipated for future studies. First, in order to validate the LES with experimental data, simulations should be conducted in a spatial configuration, in contrast to the temporal setting used in the present study. Second, the FST models considered in the present study included the assumption that the drops were affected only by the filtered flow field without direct SGS effects (i.e. the baseline unfiltered-flow-field model of Part 1). Possible improvements, if necessary, may be attained by basing the FST models on a reconstruction of the DNS flow field, that is the filtered flow field with added SGS effects, 
such as the deterministic FST model of Part 1. That FST model uses the SGS variances, which have a similar form to the SGS-fluxes, and, as shown in Part 1, can be modelled in the same manner, i.e. using the Smagorinsky, gradient and scalesimilarity models. However, using the SMD model to incorporate direct SGS effects on the drops seems likely to be disastrous since those effects would be incorrectly added, thus compounding the model errors; using the GRD or SSC models appears a more promising approach.

This work was conducted at the Jet Propulsion Laboratory (JPL) of the California Institute of Technology, and was sponsored by the US Department of Energy (contract monitors were R. Danz, Headquarters and D. Hooker, Golden Center) under an agreement with the National Aeronautics and Space Administration. Computations were performed on the SGI Origin2000 at the JPL Supercomputing Center.

\section{REFERENCES}

Bardina, J., Ferziger, J. \& Reynolds, W. 1980 Improved subgrid scale models for large eddy simulation. Tech. Rep. 80-1357. AIAA.

Boivin, M., Simonin, O. \& Squires, K. 1998 Direct numerical simulation of turbulence modulation by particles in isotropic turbulence. J. Fluid Mech. 375, 235-263.

Boivin, M., Simonin, O. \& SQuires, K. 2000 On the prediction of gas-solid flows with two-way coupling using large eddy simulation. Phys. Fluids 12 (8), 2080-2090.

Clark, R., Ferziger, J. \& Reynolds, W. 1979 Evaluation of subgrid-scale models using an accurately simulated turbulent flow. J. Fluid Mech. 91, 1-16.

Crowe, C., Chung, J. \& Troutt, T. 1998 Particle mixing in free shear flows. Prog. Energy Combust. Sci. 14, 171-194.

Deutsch, E. \& Simonin, O. 1991 Large eddy simulation applied to the modelling of particulate transport coefficients in turbulent two-phase flows. In Eighth Symp. on Turbulent Shear Flows, Technical University of Munich, pp. 10-1:1-6.

Erlebacher, G., Hussaini, M., Speziale, C. \& Zang, T. 1992 Toward the large-eddy simulation of compressible turbulent flows. J. Fluid Mech. 238, 155-185.

FurEBY, C. 1996 On subgrid scale modelling in large eddy simulations of compressible fluid flow. Phys. Fluids A 8, 1301-1311.

Germano, M., Piomelli, U., Moin, P. \& Cabot, W. 1991 A dynamic subgrid-scale eddy viscosity model. Phys. Fluids A 3, 1760-1765.

Kennedy, C. \& CARpenter, M. 1994 Several new numerical methods for compressible shear layer simulations. Appl. Numer. Math. 14, 397-433.

Le ClercQ, P. \& Bellan, J. 2004 Direct numerical simulation of a transitional temporal mixing layer laden with multicomponent-fuel evaporating drops using continuous thermodynamics Phys. Fluids 16, 1884-1907.

LiLly, D. 1992 A proposed modification of the Germano subgrid-scale closure method. Phys. Fluids A 4, 633-635.

Martin, M., Piomelli, U. \& Candler, G. 2000 Subgrid-scale models for compressible large-eddy simulations. Theor. Comput. Fluid Dyn. 13, 361-376.

MashaYeK, F. 1998a Direct numerical simulations of evaporating droplet dispersion in forced low-Mach-number turbulence. Intl J. Heat Mass Transfer 41, 2601-2617.

MashaYeK, F. $1998 b$ Droplet-turbulence interactions in low-Mach-number homogeneous shear two-phase flows. J. Fluid Mech. 367, 163-203.

MASHAYEK, F. \& JABERI, F. 1999 Particle dispersion in forced isotropic low-Mach-number turbulence. Intl J. Heat Mass Transfer 42, 2823-2836.

MilleR, R. \& Bellan, J. 1999 Direct numerical simulation of a confined three-dimensional gas mixing layer with one evaporating hydrocarbon-droplet laden stream. J. Fluid Mech. 384, 293-338.

Miller, R. \& Bellan, J. 2000 Direct numerical simulation and subgrid analysis of a transitional droplet laden mixing layer. Phys. Fluids 12, 650-671. 
Moser, R. \& Rogers, M. 1991 Mixing transition and the cascade to small scales in a plane mixing layer. Phys. Fluids A 3,1128-1134.

OKong'o, N. \& BeLlan, J. 2000 A priori subgrid analysis of temporal mixing layers with evaporating droplets. Phys. Fluids 12, 1573-1591.

OKong'o, N. \& Bellan, J. 2004 Consistent large-eddy simulation of a temporal mixing layer laden with evaporating drops. Part 1 . Direct numerical simulation, formulation and a priori analysis. J. Fluid Mech. 499, 1-47.

Poinsot, T. \& Lele, S. 1992 Boundary conditions for direct simulations of compressible viscous flows. J. Comput. Phys. 101, 104-129.

Pruett, C. \& Adams, N. 2000 A priori analyses of three subgrid-scale models for one-parameter families of filters. Phys. Fluids 12, 1133-1142.

RÉVEILlon, J. \& VervisCH, L. 2000 Spray vaporization in nonpremixed turbulent combustion modelling: A single drop model. Combust. Flame 121, 75-90.

SAnkaran, V. \& Menon, S. 2002 LES of spray combustion in swirling Flows. J. Turbulence 3 (11), $1-23$.

Simonin, O., Deutsch, E. \& Borvin, M. 1995 Large eddy simulation and second-moment closure of particle fluctuating motion in two-phase turbulent shear flows. Turbulent Shear Flows 9. Selected Papers from the 9th Intl Symp. on Turbulent Shear Flows, Kyoto, Japan, 16-18 August 1993 (ed. F. Durst, N. Kasagi, B. E. Launder et al.), pp. 85-115. Springer.

SMAGORINKSY, J. 1993 Some historical remarks on the use of nonlinear viscosities. In Large Eddy Simulation of Complex Engineering and Geophysical Flows (ed. B. Galperin \& S. Orszag), chap. 1, pp. 3-36. Cambridge University Press.

UijttewaAl, W. \& Oliemans, R. 1996 Particle dispersion and deposition in direct numerical and large eddy simulations of vertical pipe flows. Phys. Fluids 8, 2590-2604.

Vreman, B., Geurts, B. \& Kuerten, H. 1997 Large-eddy simulation of the turbulent mixing layer. J. Fluid Mech. 339, 357-390.

WANG, Q. \& SQuires, K. 1996 Large eddy simulations of particle-laden turbulent channel flow. Phys. Fluids 8, 1207-1223.

Williams, F. 1965 Combustion Theory. Addison-Wesley.

Yamamoto, Y., Potthoff, M., Tanaka, T., Kajishima, T. \& Tsuji, Y. 2001 Large-eddy simulation of turbulent gas-particle flow in a vertical channel: Effect of considering inter-particle collisions. J. Fluid Mech. 442, 303-334.

Yoshizawa, A. 1986 Statistical theory for compressible turbulent shear flows, with the application to subgrid modelling. Phys. Fluids 29, 2152-2164.

Yuu, S., Ueno, T. \& Umekage, T. 2001 Numerical Simulation of the high Reynolds number slit nozzle gas-particle jet using subgrid-scale coupling large eddy simulation. Chem. Engng Sci. 56, 4293-4307. 